\title{
Optical Properties of Isolated and Covalent Organic Framework- Embedded Ruthenium Complexes
}

\author{
Published as part of The Journal of Physical Chemistry virtual special issue "Leo Radom Festschrift". \\ Francesco Muniz-Miranda, ${ }^{\dagger, \perp, \|}$ Liesbeth De Bruecker, ${ }^{\dagger, \|}$ Arthur De Vos, ${ }^{\dagger}$ Flore Vanden Bussche, ${ }^{\ddagger, \S}$ \\ Christian V. Stevens, ${ }^{\ddagger}$ Pascal Van Der Voort, ${ }^{\S}$ (이 Kurt Lejaeghere, ${ }^{*}{ }^{\dagger}$ (i) \\ and Veronique Van Speybroeck*, ${ }^{\dagger}$ (1) \\ ${ }^{\dagger}$ Center for Molecular Modeling (CMM), Ghent University, Technologiepark 46, 9052 Zwijnaarde, Belgium \\ ${ }^{*}$ Research Group SynBioC, Department of Green Chemistry and Technology, Faculty of Bioscience Engineering, Ghent University, \\ Campus Coupure, Coupure Links 653 bl. B, 9000 Gent, Belgium \\ ${ }^{\S}$ Center for Ordered Materials, Organometallics and Catalysis (COMOC), Department of Inorganic and Physical Chemistry, Ghent \\ University, Krijgslaan 281 (S3), 9000 Gent, Belgium
}

\section{Supporting Information}

\begin{abstract}
Heterogenization of $\mathrm{RuL}_{3}$ complexes on a support with proper anchor points provides a route toward design of green catalysts. In this paper, $\mathrm{Ru}$ (II) polypyridyl complexes are investigated with the aim to unravel the influence on the photocatalytic properties of varying nitrogen content in the ligands and of embedding the complex in a triazine-based covalent organic framework. To provide fundamental insight into the electronic mechanisms underlying this behavior, a computational study is performed. Both the ground and excited state properties of isolated and anchored ruthenium complexes are theoretically investigated by means of density functional theory and time-dependent density functional theory. Varying the ligands among

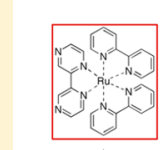

Isolated $\uparrow$ complex

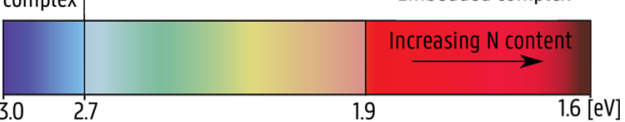
2,2'-bipyridine, 2,2'-bipyrimidine, and 2,2'-bipyrazine allows us to tune to a certain extent the optical gaps and the metal to ligand charge transfer excitations. Heterogenization of the complex within a CTF support has a significant effect on the nature and energy of the electronic transitions. The allowed transitions are significantly red-shifted toward the near IR region and involve transitions from states localized on the CTF toward ligands attached to the ruthenium. The study shows how variations in ligands and anchoring on proper supports allows us to increase the range of wavelengths that may be exploited for photocatalysis.
\end{abstract}

\section{INTRODUCTION}

Catalysis is of paramount relevance to many chemical reactions that could not occur within a reasonable time otherwise, thus disrupting either their industrial use ${ }^{1}$ or biological functionality. ${ }^{2}$ In the case of photocatalysis, the activation is effected by visible photons. This enables the synthesis of chemical products exploiting an easily available source of energy in mild reaction conditions, as often even the light emitted by a household bulb can be enough. Furthermore, control of the light exposure allows photocatalysis to be fine-tuned and even stopped at will, creating the ability to obtain a high degree of chemoselectivity. ${ }^{3,4}$ However, in the attempt to design new green catalytic routes, there is a high interest in developing recyclable and reusable heterogeneous photocatalytic systems. $^{5-8}$ One way to achieve this goal is the anchoring of homogeneous complexes with photocatalytic properties on a heterogeneous stable support. This procedure is applicable in case the photocatalytic properties of the pristine complex are retained or improved in the heterogenized system. To this end, a deep knowledge is required of both electronic and excited state properties of the pristine homogeneous and anchored complex within the support. Such investigation is presented in this paper for $\mathrm{Ru}(\mathrm{II}) \mathrm{L}_{3}$ complexes, which are commonly applied visible light photocatalysts consisting of a $\mathrm{Ru}^{2+}$ ion that is octahedrally chelated by three bidentate polypyridyl ligands as shown in Figure 1, parts a and b. Herein the electronic properties of such isolated and anchored ruthenium polypyridyl complexes onto a stable heterogeneous support are investigated.

Complexes of the type $\mathrm{Ru}\left(2,2^{\prime} \text {-bipyrdine }\right)_{3}{ }^{2+}$ are among the most interesting photoredox catalyzers. They show no catalytic properties in their ground state, but when exposed to light, their excited electronic states are able to oxidize or reduce substrate molecules through single-electron transfer (SET) processes. ${ }^{10}$ They have proven their usefulness allowing very different reactions such as carbon dioxide reduction, ${ }^{11,12}$ solar

Received: May 31, 2019

Revised: July 19, 2019

Published: July 19, 2019 


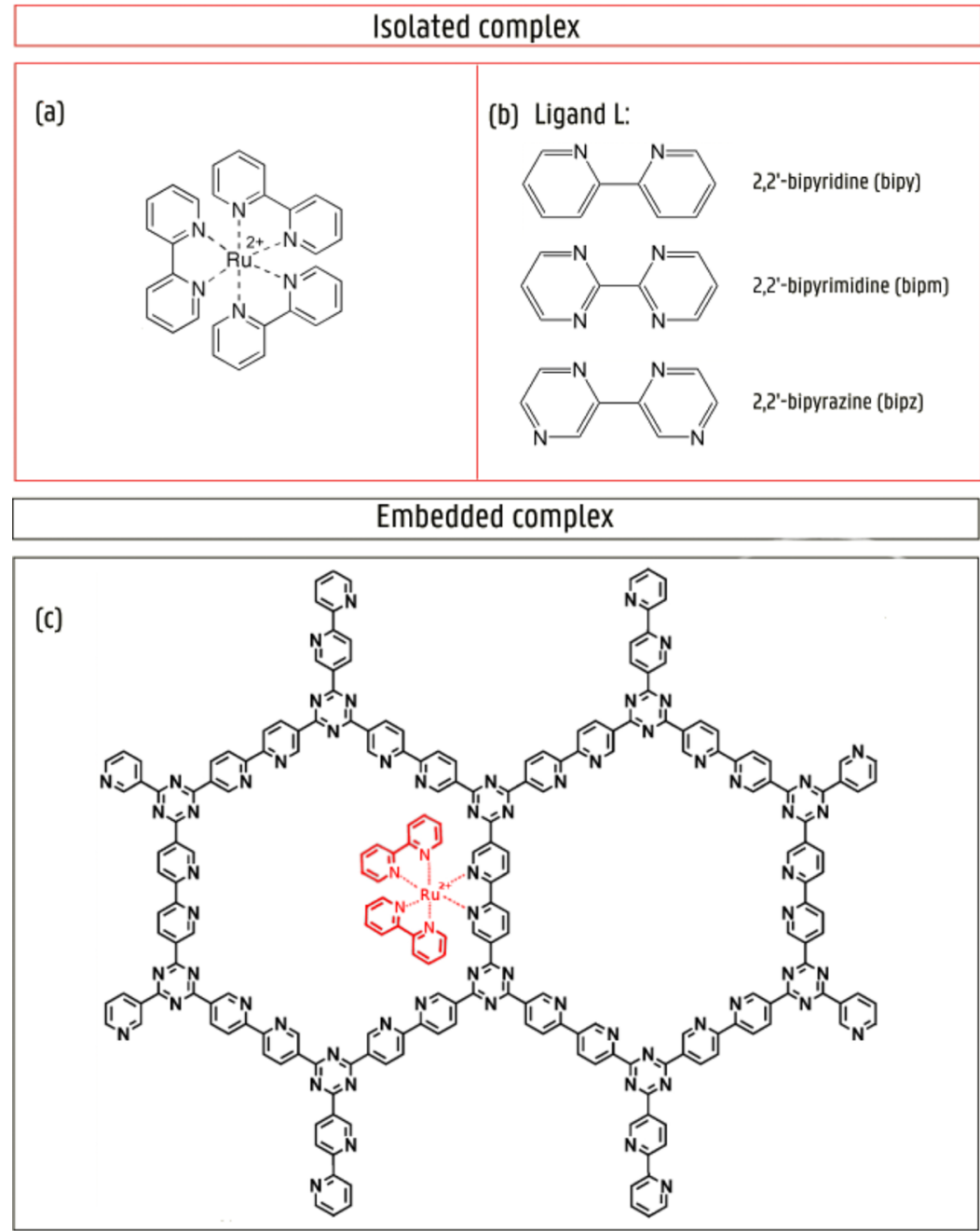

Figure 1. (a): Schematic representation of the isolated $\mathrm{Ru}(\text { bipy })_{3}{ }^{2+}$ complex. (b): Polypyridyl ligands: cis-2,2'-bipyridine (bipy), 2,2'-bipyrimidine (bipm), and cis-2,2'-bipyrazine (bipz) considered in this study. (c) Part of the CTF synthesized in ref 9 and used as computational model here, in black. The anchored $\mathrm{RuL}_{2}^{2+}$ fragment inside one pore is shown in red.

cells development, ${ }^{13,14}$ water splitting, ${ }^{15-17}$ as well as DielsAlder cycloadditions, ${ }^{18,19}$ and can be used as a photosensitizer, $^{20}$ thus spanning both inorganic and organic reactions and acting as either reducing or oxidizing agents. $^{3,21-25}$

The two possible relaxation mechanisms of the excited states of the $\mathrm{Ru}(\mathrm{II})$ cation are schematically shown in Scheme 1, which sketches the orbital occupancy of the frontier electron states of the ruthenium complex. The $t_{2 g}$ and $e_{g}$ states originate from the octahedrally surrounded $\mathrm{Ru}$ (II) cation, while orbitals from the ligands are situated in between. In the ground state, depicted on the left, only the $t_{2 g}$ states are filled and ruthenium is in the $\mathrm{Ru}$ (II) state. By absorption of a photon in the visible region a singlet $\rightarrow$ singlet metal-to-ligand charge transfer (MLCT) excitation occurs, leaving an electron hole in the metal states. As such, the oxidation number of ruthenium increases by one. The singlet excited state can quickly convert to a lower-energy triplet excited state via nonradiative means. This mechanism is called intersystem crossing (ISC). In a recent theoretical study the decay from the singlet to triplet states was shown to occur with a time constant of $26 \pm 3 \mathrm{fs}$, which was in very good agreement with experimental data. To this end a newly implemented combination of linear response time-dependent density functional theory (TD-DFT) with surface-hopping including arbitrary couplings (SHARC) was employed for the first time to model the relaxation dynamics of $\mathrm{Ru}$ (bipyridine) ${ }_{3}{ }^{2+}$ after light irradiation with explicit nonadiabatic and spin-orbit couplings. ${ }^{26}$ The resulting excited state, shown in the red box in Scheme 1, is long-lived, as the decay to the singlet ground state is spin-forbidden.

The photoexcited state can then relax either by reduction of an organic substrate, maintaining an oxidized $\mathrm{Ru}(\mathrm{III})$ cation (upper arrow in Scheme 1), or by reduction of the complex via transfer of an electron from the substrate to the now vacant $t_{2 g}$ orbital of the $\mathrm{Ru}(\mathrm{III})$ cation, which then reverts to $\mathrm{Ru}(\mathrm{II})$ (lower arrow in Scheme 1).

The triplet excited state of $\mathrm{Ru}\left(2,2^{\prime} \text {-bipyridine }\right)_{3}{ }^{2+}$ may thus be engaged in an electron transfer process, but it may also be involved in a process called triplet-triplet energy transfer (TTET), in which the decay from the excited $\mathrm{Ru}\left(2,2^{\prime}\right.$ bipyridine $)_{3}{ }^{2+}$ from its triplet to ground state involves the excitation of another molecule from its ground singlet state to its lowest triplet state. This mechanism requires the excitation of both the catalyst and substrate to a spin-triplet state and has been the subject of both theoretical ${ }^{27}$ and application-oriented investigations. ${ }^{28}$ The $\mathrm{Ru}\left(2,2^{\prime}\right.$-bipyridine $){ }_{3}{ }^{2+}$ has been exploited in a number of $\mathrm{C}-\mathrm{C}$ making and breaking transformations, such as trans/cis stilbene isomerization, ${ }^{29}$ anthracene dimeri- 
Scheme 1. Photoactivation of an Octahedral Ruthenium Complex from the Ground State (Left Black Box) to an Excited State (Red Box) under the Influence of Visible Light, Exciting an Electron toward a State Localized on the Ligands, followed by ISC $^{a}$

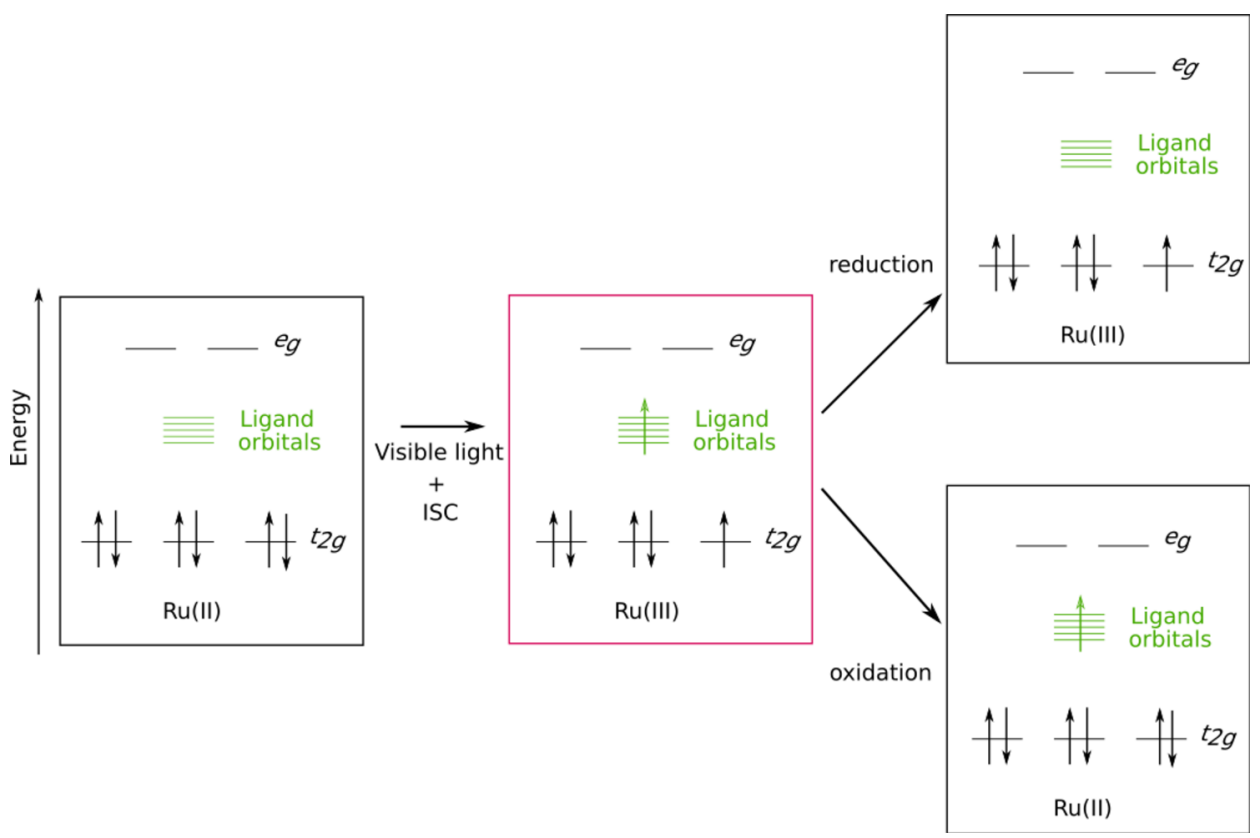

${ }^{a_{T}}$ The excited state relaxes by reduction (upper arrow) or oxidation (lower arrow) of the substrate. Schematic adapted from ref 3 . Copyright 2013 American Chemical Society.

zation, ${ }^{30}$ cycloadditions, ${ }^{31}$ and trifluoromethylations of styrene substrates $^{32}$ via the TTET mechanism.

Herein we investigate the photocatalytic properties of $\mathrm{Ru}(\mathrm{II}) \mathrm{L}_{3}$ type complexes, where the ligands may either be cis-2,2'-bipyridine (bipy), 2,2'-bipyrimidine (bipm), or cis-2,2'bipyrazine (bipz) as schematically shown in Figure $1 \mathrm{~b}$. Complexes with a varying number of nitrogen containing aromatic rings have been synthesized both in the literature $^{33-35}$ and in this work. In the first instance, we investigate to what extent the nitrogen content affects the photocatalytic properties. Second, the impact of heterogenizing the $\mathrm{Ru}(\mathrm{II}) \mathrm{L}_{3}$ complexes on covalent triazine frameworks (CTFs) (see Figure 1c), which are a subclass of the broader family of covalent organic frameworks (COFs), are assessed. ${ }^{36}$ Unfunctionalized COFs have been explored within catalysis to some extent, ${ }^{6,37-39}$ but additional functionalization of the materials with metal complexes may open additional perspectives for their usage within catalysis by merging some of the most important features of both the organic, i.e., stability, and the inorganic, i.e., catalytic properties, worlds.

CTFs are potentially interesting supports since they are chemically and thermally stable. ${ }^{40-46}$ Furthermore, they are much lighter than most other porous supports and contain no toxic or environmentally unfriendly elements. These porous 2D materials are made by ionothermal trimerization of aromatic nitriles and when fabricated with bidentate nitrogen containing ligands-similar to the chelating ligands in the ruthenium homogeneous complex - they may serve as ideal anchoring materials for the latter complexes. ${ }^{47}$ The first reports on COFs containing (bi)pyridine have appeared recently, showing great promise for applications in catalysis and gas sorption. ${ }^{9,48-54}$ In a similar way, this has already been applied for metal organic frameworks (MOFs), ${ }^{55}$ where photocatalytic complexes were successfully anchored to both linkers ${ }^{56,57}$ and nodes. ${ }^{58}$ However, MOFs are in general less stable, making it interesting to explore other heterogeneous supports. ${ }^{59-61}$

To optimize the photocatalytic system, a thorough understanding of the ground- and excited-state properties of the tethered $\mathrm{RuL}_{3}{ }^{2+}$ complexes is mandatory. ${ }^{62}$ Ground state properties of the heterogeneous system have been studied in detail in our previous work, ${ }^{63}$ whereas in this work, we focus on the calculation of excited states. The investigation of the distribution of most singlet states can be performed experimentally by UV-vis absorption spectroscopy. Contrarily, triplet states are more difficult to characterize without a computational investigation due to electronic selection rules preventing singlet $\rightarrow$ triplet excitations to achieve a significant oscillator strength, i.e., probability of occurrence. In order to understand how the CTF support impacts the photocatalytic properties of the ruthenium complexes, a stepwise computational analysis is adopted. The ground- and excited-state electronic properties of both the isolated ruthenium complexes and the anchored systems are investigated by means of density functional theory (DFT) and time-dependent density functional theory (TD-DFT).

To this end, we embed $\mathrm{RuL}_{2}{ }^{2+}$ (represented in red in Figure 1c) into an extensive CTF structure model (represented in black in Figure 1c). ${ }^{9}$ This CTF organic scaffold contains bipyridine groups belonging to the CTF exposed inside the pores, which are suitable to act as linkers for the ruthenium complex, thus giving rise to a $\mathrm{RuL}_{2}{ }^{2+} @ \mathrm{CTF}$ compound. The results are analyzed to obtain insight into the electronic charge rearrangement and optical gap tuning induced by the ligands. For the isolated ruthenium complexes, we are able to compare and validate the calculated optical properties with experimentally measured UV-vis absorption data. However, specifically investigating the excitations of COFs by means of $\mathrm{UV}$-vis absorption spectroscopy is cumbersome as these 
compounds often absorb light in a wide wavelength region, in part due their high flexibility. In addition, photoemission spectroscopy may yield results difficult to interpret without some theoretical understanding. In this light the current computational approach gives new insights into the excited state properties of the $\mathrm{RuL}_{2}{ }^{2+} @ \mathrm{CTF}$ system, which in turn affect its photocatalytic properties. While TD-DFT calculations have been performed on COFs before, ${ }^{64}$ to our knowledge this is one of the first applications on a COF model to which a photoactive complex has been anchored.

\section{COMPUTATIONAL DETAILS}

All calculations were performed with the Gaussian 16 software. ${ }^{65}$ Ground state information was extracted at the DFT level. Excited states were investigated adopting the TDDFT scheme, ${ }^{66}$ within the linear-response approach due to Casida. $^{67}$

2.1. Assessment of the Level of Theory for the Ground States. Four different exchange-correlation functionals coming from different rungs on Jacob's ladder ${ }^{68}$ were tested on isolated $\mathrm{RuL}_{3}{ }^{2+}$ complexes to compare their relaxed structures: the generalized gradient approximation $\mathrm{PBE}{ }^{69}$ hybrid B3LYP, ${ }^{70,71}$ long-range corrected CAM-B3LYP, ${ }^{72}$ and metahybrid M06. ${ }^{73}$ All computed internal normal modes of the relaxed structures show positive frequencies, ensuring that the optimized geometries represent minima of the ground state potential energy surface.

The average differences in bond lengths calculated with the various functionals and basis sets are below the $0.05 \AA$ threshold. The optimized structures for $\mathrm{Ru}(\text { bipy })_{3}{ }^{2+}$ are included in section S4 of the Supporting Information. All levels of theory employed here predict a positive partial Hirshfeld charge on the $\mathrm{Ru}(\mathrm{II})$ cations, with differences between the same complexes of about $0.02|e|$, with $e$ the electron charge (see Table S1 of the Supporting Information).

2.2. Assessment of the Level of Theory for the Excited States. The vertical excitation spectra from the ground state geometry of $\mathrm{Ru}(\text { bipy })_{3}{ }^{2+}$, obtained by $\mathrm{TD}$ DFT, ${ }^{67,74}$ are shown in Figure 2. Theoretical calculations were carried out both in the gas phase, i.e., without solvent, and with water and acetonitrile solvents modeled with the polarizable continuum method (PCM) to take into account the effect of the environment on the excitation energies. ${ }^{75}$ The simulated $\mathrm{UV}$-vis absorption spectra at the TD-DFT level of theory are compared with the experimental optical profiles for $\mathrm{Ru}$ (bipy) ${ }_{3}^{2+}$ complexes in water $^{76}$ and acetonitrile solvents. The latter experimental data were generated within the framework of this work (vide infra for details on the experimental part). A side note is warranted on how to compare the theoretical and experimental data. The TD-DFT energy of the first allowed transition is an approximation of the optical gap, which is defined by a neutral excitation and as the difference between the energies of the lowest dipole-allowed excited state and the ground $^{\text {state. }}{ }^{77}$ As it accounts for the electron-hole recombination energy or exciton binding energy, the optical gap is systematically lower than the fundamental gap, ${ }^{78}$ defined by a charged excitation and as the difference between the first ionization potential and the first electron affinity. ${ }^{77}$ From UVvis absorption measurements, optical gaps can be extrapolated as the energy of the first divergence of the spectrum from its baseline.

The spectrum calculated with the M06 functional yields the best agreement with experimental data with respect to those

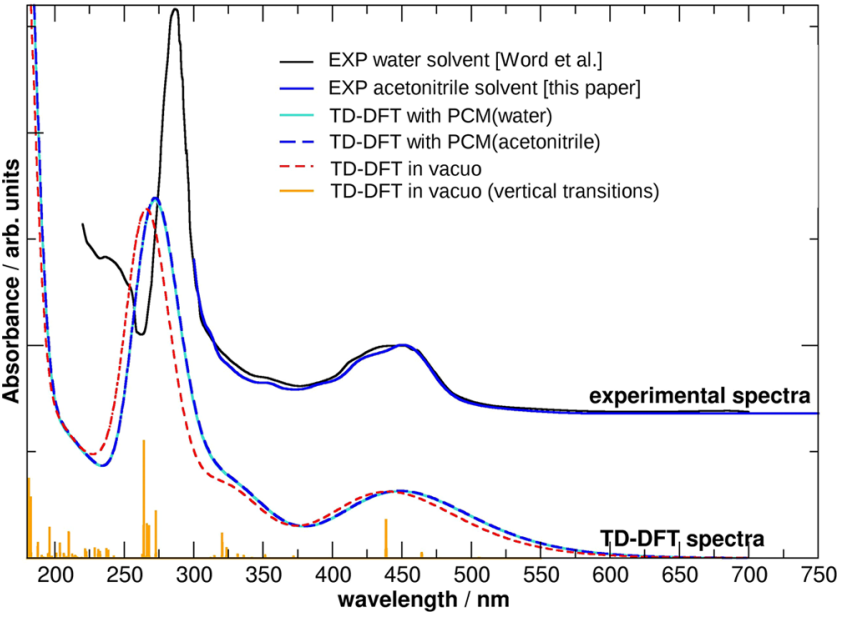

Figure 2. Experimental UV-vis absorption (top, Word et al. ${ }^{76}$ and this paper) and TD M06 spectra (bottom) of Ru(bipy) ${ }_{3}{ }^{2+}$. Vertical excitations for the in vacuo TD M06 spectrum are reported as orange spikes. Computed spectra have been smoothed using Gaussian functions of half-width at half-height of $0.333 \mathrm{eV}$ (default value of Gaussview). The computed spectra have not been rescaled nor shifted.

computed with PBE, B3LYP, and CAM-B3LYP (see Figure S1 of the Supporting Information) in terms of both shapes and positions of the bands, and no further rescaling of energies nor of wavelengths - as often employed for similar comparisons when pseudopotentials are used to simulate core electrons in metal atoms-were necessary. ${ }^{79}$ As expected, ${ }^{80,81}$ TD PBE spectra obtained by using a semilocal functional without Hartree-Fock exchange necessitated significant blueshifts to match the experimental data. Contrarily, TD CAM-B3LYP spectra required a significant redshift, in agreement with earlier studies on compounds ranging from metal-organic frameworks (MOFs) ${ }^{82}$ to noble metal nanoclusters. ${ }^{80}$ TD B3LYP spectra are rather similar to TD M06 spectra in frequencies, but the agreement regarding the shape of the absorption bands is better when using the M06 functional. As the M06/ LanL2DZ level of theory correctly reproduces the optical features of the studied complexes, we have consistently used this level of theory unless otherwise stated. Errors between the various tested functionals are always smaller than $20 \mathrm{~nm}$ (see Figure $S 1$ of the Supporting Information). We used the LanL2DZ ${ }^{83}$ basis as it has been shown that the choice of basis set has a minimal influence on excitation energies, oscillator strengths, and assignments for transition metal complexes. ${ }^{84}$ Tests with the Def2TZVPP basis set reveal a difference for the energy of the strongest transition of only $11 \mathrm{~nm}$. Overall, M06 performs particularly well in systematic reviews of both organic and inorganic molecules, ${ }^{85,86}$ yielding accurate transition energies.

Our conclusions are in agreement with $\mathrm{ref}^{87}$ in which several functionals were tested for a few ruthenium complexes, including $\mathrm{Ru}$ (bipy) ${ }_{3}{ }^{2+}$. Besides the excitation energies, for which hybrid functionals and their range-separated and meta counterparts are the most accurate, Atkins et al. focused on the energy gaps between excited states, which tend to be best described by the pure generalized gradient approximation exchange-correlation functionals. ${ }^{87}$ Since we are in this work interested in excitation energies, this confirms our choice for the M06 functional. 

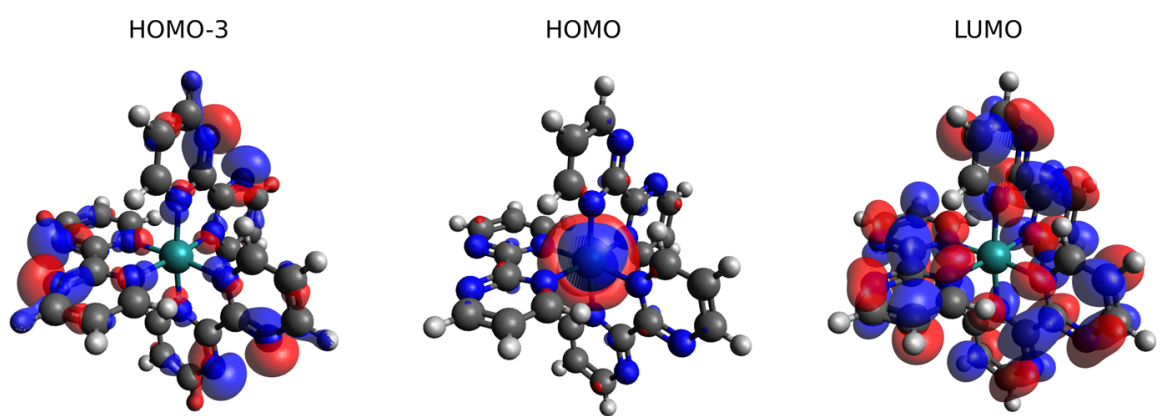

Figure 3. Isosurfaces of the $\mathrm{HOMO}-3, \mathrm{HOMO}$, and LUMO of the $\mathrm{Ru}(\mathrm{bipm})_{3}{ }^{2+}$ compound (complex g in Figure 4). Calculation carried out at the M06/LanL2DZ level of theory.
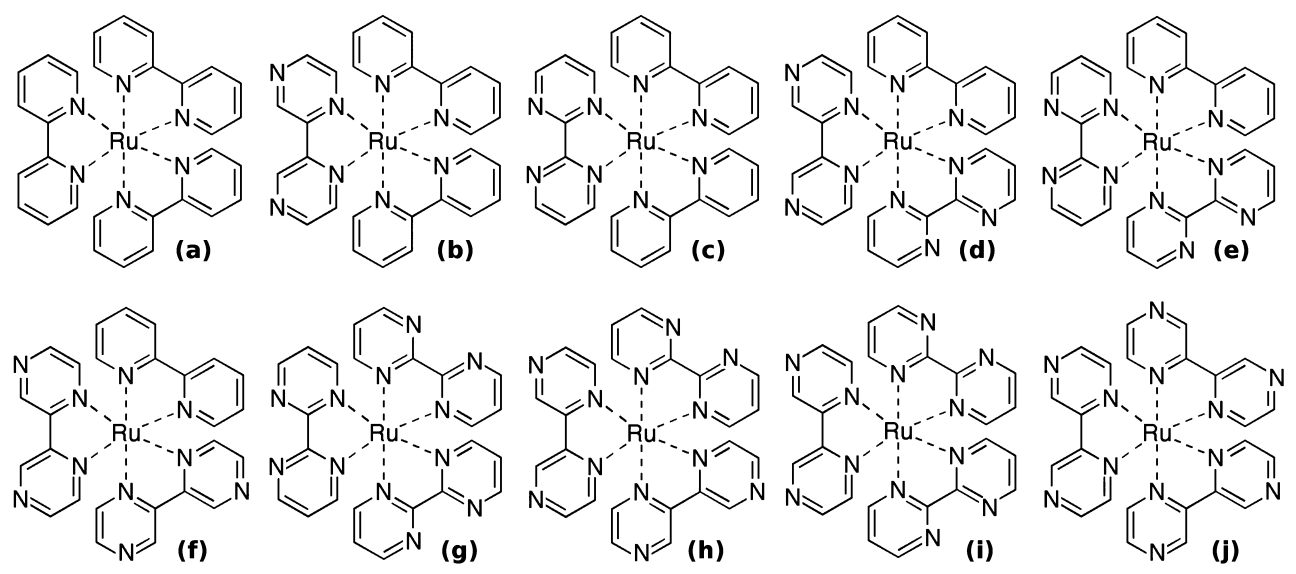

Figure 4. Ten $\mathrm{RuL}_{3}{ }^{2+}$ complexes investigated here. (a) $\mathrm{L}=$ bipy $\times 3$; (b) $\mathrm{L}=$ bipy $\times 2$, bipz $\times 1$; (c) $\mathrm{L}=$ bipy $\times 2$, bipm $\times 1$; (d) $\mathrm{L}=$ bipy $\times 1$, bipz $\times 1$, bipm $\times 1$; (e) $\mathrm{L}=$ bipy $\times 1$, bipm $\times 2$; (f) $\mathrm{L}=$ bipy $\times 1$, bipz $\times 2$; $(\mathrm{g}) \mathrm{L}=\operatorname{bipm} \times 3$; (h) $\mathrm{L}=\operatorname{bipz} \times 2$, bipm $\times 1$; (i) $\mathrm{L}=$ bipz $\times 1$, bipm $\times 2$; (j) $\mathrm{L}=$ bipz $\times 3$.

The overall shape of the spectrum is due to the many transitions from the singlet ground state to singlet excited states $\left(S_{0} \rightarrow S_{n} ; n=1, \ldots\right)$, which are spin-allowed. The effect of the solvent seems very modest, both from an experimental and a computational point of view. In addition, the effect of PCM with respect to the calculation in vacuo (dashed red line in Figure 2) is negligible for the first strong absorption band centered at about $\sim 450 \mathrm{~nm}(\sim 2.75 \mathrm{eV})$ and is rather small for the second peak located between 300 and $200 \mathrm{~nm}$, i.e., in the UV region, with discrepancies smaller than $10 \mathrm{~nm}$.

2.3. Optimization of the Ru(II)@CTF Models. To investigate the influence of embedding the ruthenium complexes within a CTF support, a Ru(II)@CTF model was constructed as schematically shown in Figure 1c. A CTF composed of triazine nodes interlinked with bipy linkers was considered. The bipy residues embedded into the CTF can naturally act as ligands for the ruthenium complexes, together with two other ligands that remain exposed inside the pore of the CTF.

The investigated model consists of two full CTF pores to minimize "edge effects" due to the sudden truncation of our cluster model (see Figure 1c). The cluster was terminated by completing the coordination of carbon atoms with hydrogen atoms. During the geometry optimization, the structures were allowed to relax freely and they converged to approximately planar structures, with small differences due to the different types of ligands.

Because the models including the COFs are very large (10 triazine and 34 pyridine rings, see Figure 1c), geometry optimizations of the models including the COFs were carried out with a step-by-step approach. First, the COFs were optimized using a small basis set (e.g., LanL1DZ). Second, the system was reoptimized with the addition of the $\mathrm{Ru}$ (II) cation and the two other ligands at the same level of theory. Finally, the total system was reoptimized at the M06/LanL2DZ level of theory within Gaussian 16 standard convergence criteria.

\section{EXPERIMENTAL DETAILS}

The UV-Vis absorption spectrum was collected using a Shimadzu UV1800 UV-vis spectrometer. The sample was prepared by dissolving $1 \mathrm{mg}$ of $\mathrm{Ru}(\text { bipy })_{3}\left(\mathrm{PF}_{6}\right)_{2}$ (SigmaAldrich) in $1.5 \mathrm{~mL}$ acetonitrile (Sigma-Aldrich, used without further purification). The solution was filtered through a 0.45 $\mu \mathrm{m}$ Whatman syringe filter and placed in a $1 \mathrm{~cm} \times 1 \mathrm{~cm}$ cuvette. Further dilution of the sample was performed to obtain the desired absorbance range.

\section{RESULTS AND DISCUSSION}

4.1. Ground State Properties of Isolated Complexes. The three ligands investigated here all maintain a largely planar geometry when coordinating with a $\mathrm{Ru}(\mathrm{II})$ cation; the dihedral angles linking the two aromatic subunits are less than $1.5^{\circ}$. However, they contain a different number of nitrogen atoms (2 in bipy and 4 in bipm and bipz as shown in Figure 1b). Frontier orbitals of the ruthenium complexes may be sensitive to the nitrogen content of the ligands and their different aromaticity. In Figure 3, the HOMO and LUMO of $\mathrm{Ru}(\text { bipm })_{3}{ }^{2+}$ are displayed, showing a general trend for this class of complexes. The HOMO, which is a nonbonding (n) 


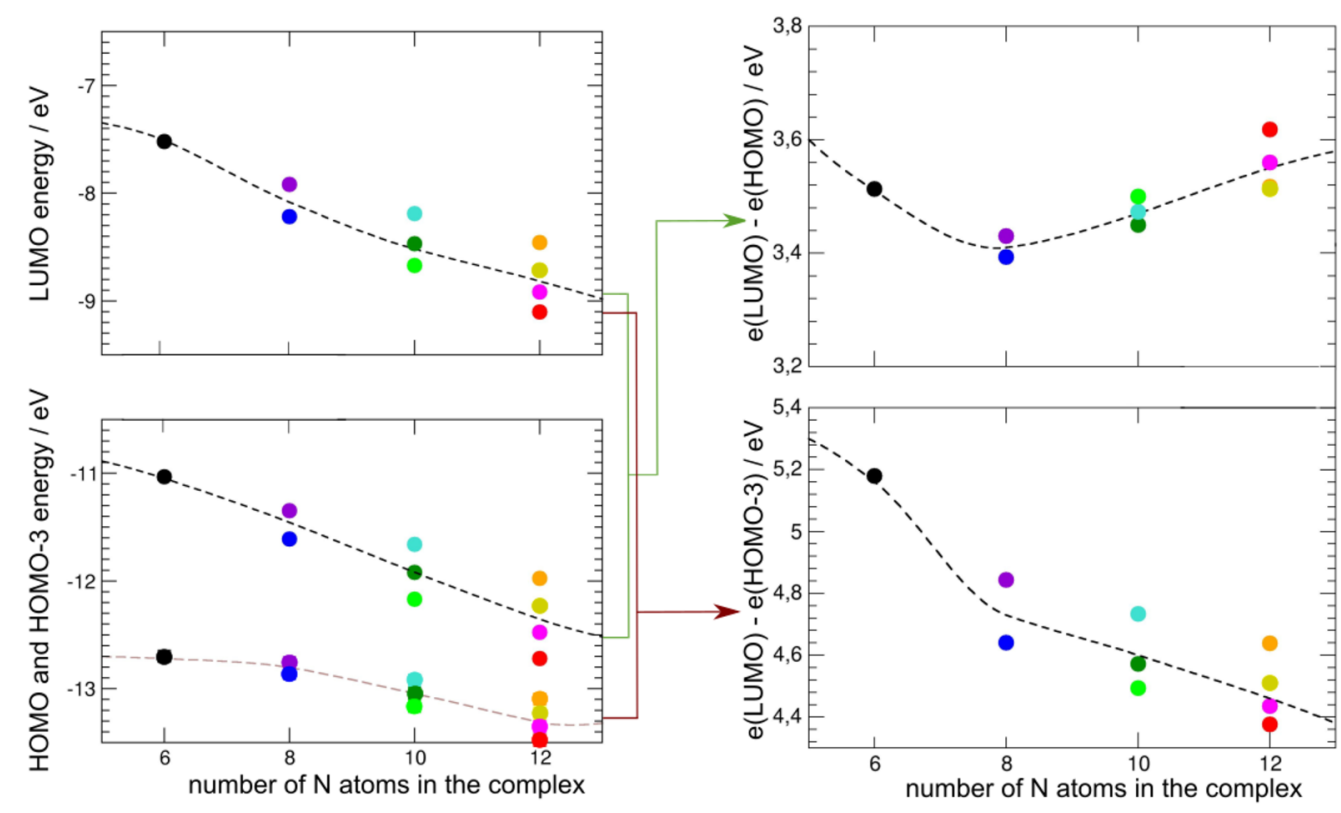

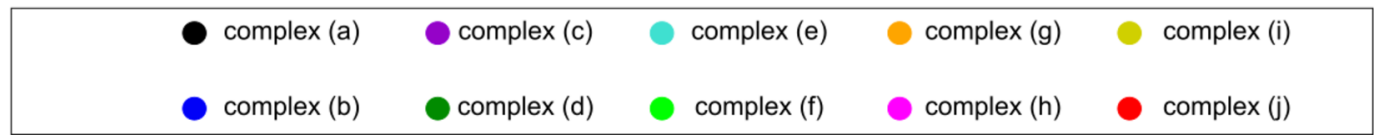

Figure 5. Energies of LUMOs (top left panel), HOMOs and HOMOs-3 (bottom left panel). Energy differences between HOMOs and LUMOs (top right panel) and HOMOs-3 and LUMOs (bottom right panel). The ten $\mathrm{RuL}_{3}{ }^{2+}$ complexes are (a) $\mathrm{L}=$ bipy $\times 3$; (b) $\mathrm{L}=$ bipy $\times 2$, bipz $\times 1$; (c) $\mathrm{L}=$ bipy $\times 2$, bipm $\times 1$; (d) $\mathrm{L}=\operatorname{bipy} \times 1$, bipz $\times 1$, bipm $\times 1$; (e) L = bipy $\times 1$, bipm $\times 2$; (f) $\mathrm{L}=$ bipy $\times 1$, bipz $\times 2$; $(\mathrm{g}) \mathrm{L}=$ bipm $\times 3$; (h) L $=\operatorname{bipz} \times 2$, bipm $\times 1$; (i) L = bipz $\times 1$, bipm $\times 2$; and $(j) L=b i p z \times 3$. Calculation carried out at the M06/LanL2DZ level of theory.

orbital, is mainly localized on the central $\mathrm{Ru}(\mathrm{II})$ cation, thus largely coinciding with a $t_{2 g}$ orbital, whereas the LUMO, which is a $\pi^{*}$ orbital, is localized on the ligands. In addition, also the HOMO-3 orbital is visualized as it is the first occupied orbital below the HOMO, which is mainly localized on the ligands. In contrast, the $\mathrm{HOMO}-1$ and $\mathrm{HOMO}-2$ orbitals are mainly of the $t_{2 g}$ type (see Figure S2 of the Supporting Information).

It is now interesting to investigate to what extent the nitrogen content in the ligands affects their orbital energies. Various complexes were considered as schematically shown in Figure 4. For each of these complexes the energies of the HOMO, LUMO, and HOMO-3 in terms of the number of nitrogen atoms are plotted in Figure 5. The energies of both frontier orbitals decrease by $\sim 1 \mathrm{eV}$ by going from the $\mathrm{Ru}(\text { bipy })_{3}{ }^{2+}$ complex, containing only 6 nitrogen atoms (black circles), to complexes with only bipz and bipm ligands (red, pink, yellow, and orange circles), which contain 12 nitrogen atoms instead. The simultaneous decrease in energy for both types of orbitals results in a rather flat profile by about $0.2 \mathrm{eV}$ for the HOMO-LUMO gaps. Nevertheless, a small minimum at $N=8$ atoms can be discerned, a recurring feature in many energy gap profiles of these complexes as will be shown later.

While the HOMO-LUMO gap seems largely unaffected by the ligands, the energy gap between orbitals mainly localized on the ligands shows a different behavior. The contribution of the nitrogen atoms is small in the HOMO-3 orbital, as can be seen in the expansion in atomic contributions of the wave function (Table S2 in Supporting Information). As a result, the energy of the HOMO-3 is only slightly affected by the increase in number of nitrogen atoms from 6 to 12 (Figure 5 bottom left panel). Then, as expected, the energy gap between the LUMO and HOMO-3, which is indicative for ligand- ligand transitions, decreases with the number of nitrogen atoms in the complexes, as shown in the bottom right panel of Figure 4. These conclusions are in agreement with our previous work on the ground state properties of $\mathrm{RuL}_{3}{ }^{2+} \cdot{ }^{2}$ Thus, the energy difference between the HOMO-3 and LUMO can be considered as a sort of effective "ligand gap", i.e., an energy gap between states mainly localized on the organic rings. Even if this gap shows significant internal variations for a fixed number of nitrogen atoms in the complex, it seems to be more clearly dependent on the nitrogen content than the HOMO-LUMO gap, as it spans a full $1 \mathrm{eV}$ range (see Figure 5, right panels).

Further clarification for the previous findings can be found by inspecting the electronic density of states (eDoS) for $\mathrm{Ru}(\text { bipy })_{3}{ }^{2+}$ (see Figure 6). It confirms that the major contributions of ruthenium around the band gap are found on the HOMO, HOMO-1, and HOMO-2.

Such an analysis was also performed for the different complexes having a varying degree of nitrogen content. The eDoSs are superimposed in the left pane of Figure 7, showing that the nitrogen content actually affects all the eigenvalues of the systems. With an increasing number of nitrogen atoms in the ligands we observe decreasing energies; i.e., the distribution is shifted to the left side of the plot, as indicated by the colored arrow. The contributions of the $\mathrm{eDoS}$ originating from ruthenium, while mainly localized on the HOMOs and on virtual states which are about $5 \mathrm{eV}$ higher in energy than the LUMOs (see Figure 6), are still affected by the number of nitrogen atoms in the complexes, as shown in the right pane of Figure 7.

The organic ligands investigated here are all heteroaromatic compounds and the degree of aromaticity might play a role to 


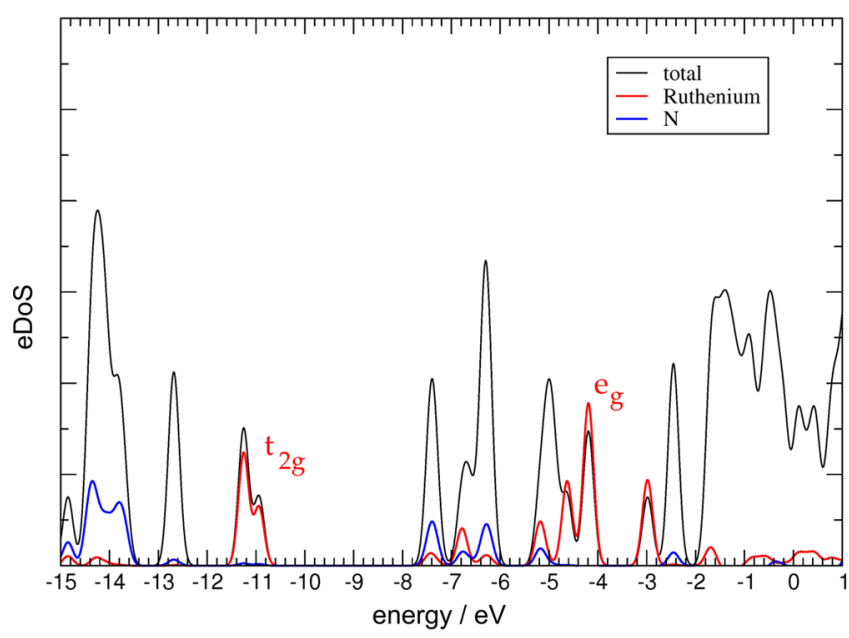

Figure 6. eDoS of $\mathrm{Ru}$ (bipy) ${ }_{3}{ }^{2+}$ complex, with contributions due to the $\mathrm{Ru}(\mathrm{II})$ cation and the $\mathrm{N}$ atoms colored in red and blue, respectively. Calculation carried out at the M06/LanL2DZ level of theory. The zero is set at the calculated ionization energy.

elucidate the observed trends for the energy levels in terms of the nitrogen content. Aromaticity is usually considered as a particular stabilization of the occupied states localized on aromatic rings with respect to the virtual states. This property can be quantified using a number of different scales and indexes. Herein we used the aromaticity index of Bird, ${ }^{88,89}$ which is based on the resonance stabilization energy. The latter property is smaller for pyrimidine $(40.6 \mathrm{kcal} / \mathrm{mol})$ and pyrazine $(40.9 \mathrm{kcal} / \mathrm{mol})$ than for pyridine $(43.3 \mathrm{kcal} /$ mol). ${ }^{88}$ This fact can be qualitatively understood in terms of electron content because adding more nitrogen atoms, with additional electron pairs that do not participate in delocalized $\pi$ orbitals, decreases the overall stabilization. We notice that the energy difference between the HOMO-3 and LUMO has some correlation with Bird's aromatic stabilization energy, as shown in Figure S3 of the Supporting Information. However, we have not found any clear correlation between the HOMOLUMO gap and the aromaticity index of Bird. ${ }^{88,89}$ This is reasonable since this gap is strongly affected by ruthenium, which is not taken into account by this aromaticity descriptor. We also investigated the correlation with some other aromaticity indices. More information can be found in section S2.1 and Figure S4 of the Supporting Information.

In order to effectively catalyze redox reactions, ruthenium has to possess a net charge. The partial charges obtained with both the Hirshfeld and Mulliken partition schemes of the ten complexes are reported in Table S3 of the Supporting Information. As can be seen, the $\mathrm{Ru}$ (II) cation is consistently positive, but a correlation with the number of nitrogen atoms or aromatic descriptors is not evident, because the differences are rather small, being of the order of 0.02 lel. As expected, the Hirshfeld charges are in absolute values smaller than the Mulliken charges. $^{90}$

In conclusion from the ground state properties of the isolated $\mathrm{Ru}(\mathrm{L})_{3}{ }^{2+}$, it is observed that the HOMO-LUMO gap-approximately describing the MLCT-remains nearly unaffected by the number of nitrogen atoms, whereas the HOMO-3-LUMO gap-approximately describing the ligand-ligand gap-is reduced for a higher number of nitrogen atoms.

4.2. Excited States Properties of Isolated Complexes. The photoredox and charge-transfer properties are strongly affected by the optical properties of the complexes, in particular by their absorption and emission of UV-vis radiation. First, we discuss the singlet and triplet excitations in the visible-light range which are mainly of the MLCT type, as stated before. Afterward, we focus on the triplet metalcentered states. The triplet excitations are spin-forbidden but play an important role in TTET. The energy of the first spinallowed transition, i.e., the energy of the first singlet state $\left(S_{1}\right)$, approximates the optical gap, as discussed previously. From our TD-DFT calculations we determine how it changes with the nitrogen content of the complexes. The results are shown in the upper panel of Figure 8.

The complex showing the lowest $S_{1}$ energy contains eight nitrogen atoms and is composed of two bipy ligands and one bipz (blue dot, complex b), whereas the complex with the highest energy is $\mathrm{Ru}(\mathrm{bipz})_{3}{ }^{2+}$ (red dot, complex $\mathrm{j}$ ), with a difference of about $0.4 \mathrm{eV}$. A similar distribution of values,
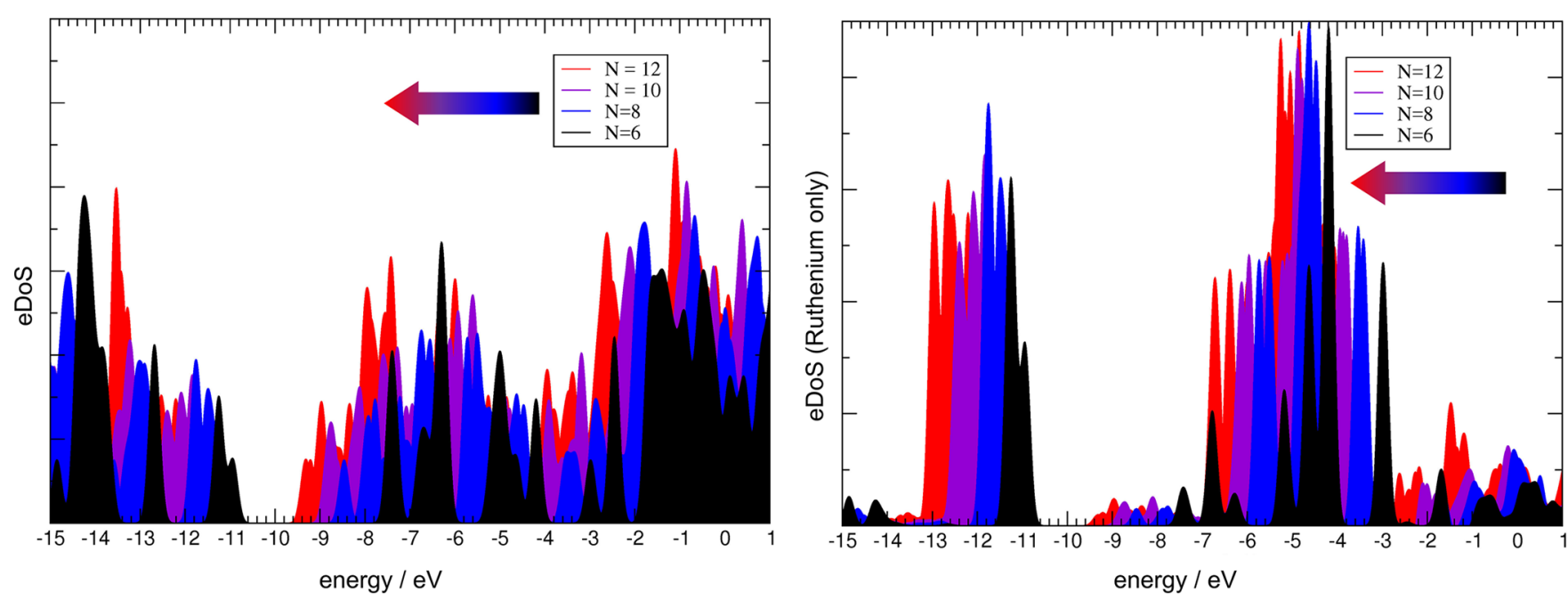

Figure 7. Total eDoS (left) and contribution localized on ruthenium (right) of the ten complexes investigated here, sorted by color according to the number of $\mathrm{N}$ atoms in the ligands $(\mathrm{N}=6,8,10$, and 12). The eDoS are superimposed. Calculations have been carried out at the M06/ LanL2DZ level of theory. 


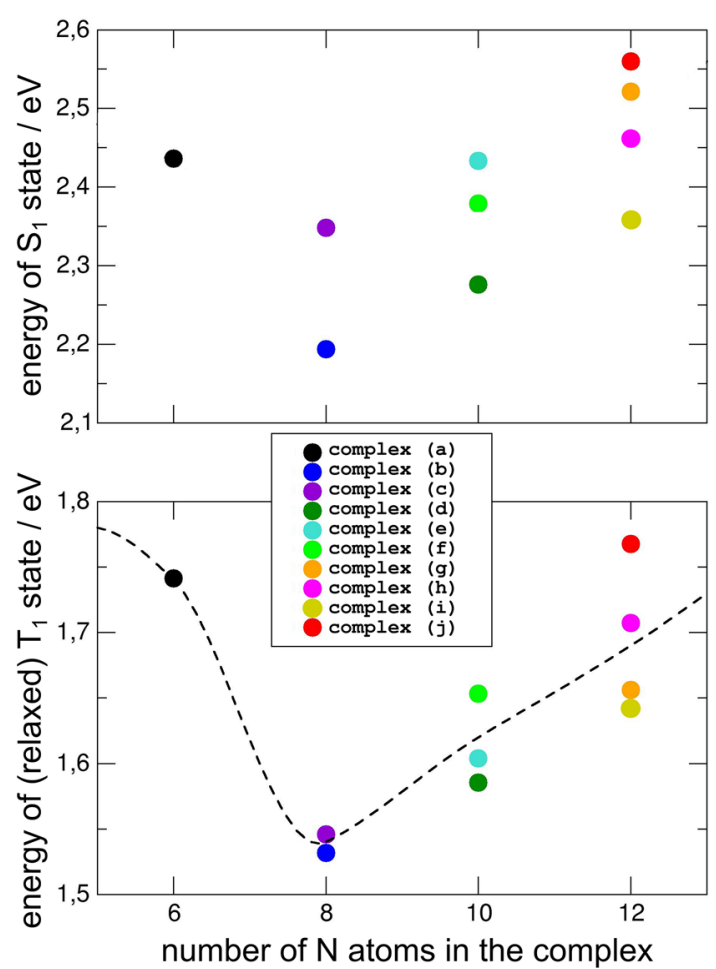

Figure 8. Vertical TD-M06 energies of the $S_{1}$ states (upper panel) and of the optimized $T_{1}$ states (lower panel). The ten $\mathrm{RuL}_{3}{ }^{2+}$ complexes are (a) $\mathrm{L}=$ bipy $\times 3$; (b) $\mathrm{L}=$ bipy $\times 2$, bipz $\times 1$; (c) $\mathrm{L}$ $=$ bipy $\times 2$, bipm $\times 1$; (d) $\mathrm{L}=$ bipy $\times 1$, bipz $\times 1$, bipm $\times 1$; (e) $\mathrm{L}=$ bipy $\times 1$, bipm $\times 2$; (f) $\mathrm{L}=$ bipy $\times 1$, bipz $\times 2$; $(\mathrm{g}) \mathrm{L}=$ bipm $\times 3$; (h) $\mathrm{L}=\operatorname{bipz} \times 2$, bipm $\times 1$; (i) $\mathrm{L}=\operatorname{bipz} \times 1$, bipm $\times 2$; and $(\mathrm{j}) \mathrm{L}=\operatorname{bipz}$ $\times 3$ according to the nomclature introduced in Figure 4. Calculation carried out at the M06/LanL2DZ level of theory.

albeit with different magnitudes, can be observed for the most intense transitions of the visible spectra, occurring between 3 and $2.7 \mathrm{eV}$, at $\sim 430 \mathrm{~nm}$ as can be seen in Figure S5 of the Supporting Information. All excitation energies and their corresponding oscillator strengths are given in Table S4 of the Supporting Information.

Although the experimental absorption spectrum is mainly due to vertical excitations, adhering to the Franck-Condon principle, we also examined to which extent the geometries of the excited MLCT $S_{1}$ and $S_{2}$ states change when we optimize them. These states correspond to adiabatic, i.e., nonvertical, excitations and may be observed in fluorescence measurements. We find that their relaxed excited-state geometries are very similar to those of their respective ground states (see Figure S6 (left pane) Supporting Information). Moreover, the overall effect of geometry optimization on the excitation energies is a shift toward lower values, which is rather constant, i.e., between 0.43 and $0.66 \mathrm{eV}$ for the ten $\mathrm{RuL}_{3}{ }^{2+}$ complexes (Table S4 and S5 of the Supporting Information). For $\mathrm{Ru}$ (bipy) ${ }_{3}{ }^{2+}$ the $\mathrm{S}_{1}$ energy for the relaxed geometry becomes $2.00 \mathrm{eV}$ (compared with $2.45 \mathrm{eV}$ when the geometry of the excited state is not optimized), corresponding to a wavelength of $618.7 \mathrm{~nm}$. This is in good agreement with a fluorescence emission occurring at $615 \mathrm{~nm}^{.91,92}$

Nested between the $S_{0} \rightarrow S_{n}$ transitions, there are spinforbidden MLCT transitions to triplet states $\left(\mathrm{T}_{n}\right)$, whose contributions to the absorption spectra are negligible. However, these $T_{n}$ states play a role in TTET and can still be encountered during nonradiative processes and decays which require a relaxation of the geometry. They therefore have to be taken into account to describe the electronic structures of complexes with emerging catalytic properties. ${ }^{93,94}$ The adiabatic transition energies of the first triplet states closely follow the trend of the $S_{1}$ ones (Figure 8, bottom panel), albeit with a smaller overall variation $(\sim 0.3 \mathrm{eV}$ instead of $0.4 \mathrm{eV}$ ) and at lower energies. However, they still pinpoint $\mathrm{Ru}(\text { bipy })_{2}(\mathrm{bipz})^{2+}$ as the complex with the smallest transition energy $(N=8)$. As expected, the distributions of $T_{1}$ and $S_{1}$ excitation energies versus the number of nitrogen atoms also follow a pattern very similar to that of the HOMO-LUMO gaps (see Figure 5).

Transitions toward $\mathrm{T}_{1}, \mathrm{~T}_{2}$, and $\mathrm{T}_{3}$ states are all of the MLCT type. In the case of the $\mathrm{Ru}$ (bipy) ${ }_{3}{ }^{2+}, \mathrm{Ru}(\mathrm{bipz})_{3}{ }^{2+}$, and $\mathrm{Ru}(\mathrm{bipm})_{3}{ }^{2+}$ complexes, i.e., those with three equal ligands, the corresponding vertical transitions are basically degenerate as the energy difference is below the $0.02 \mathrm{eV}$ threshold. This degeneracy is removed when a ligand is substituted with one of a different type, as shown in Figure S7 of the Supporting Information for the case of $\mathrm{Ru}$ (bipy) ${ }_{3}{ }^{2+}$. By a subsequent exchange of ligands, the $\mathrm{T}_{1}-\mathrm{T}_{2}-\mathrm{T}_{3}$ energy separation increases from less than $0.02 \mathrm{eV}$ to more than $0.2 \mathrm{eV}$. In addition, the triplet transitions whose degeneracy is broken by passing from $\mathrm{RuL}_{3}{ }^{2+}$ to a $\mathrm{Ru}(\mathrm{L})_{2} \mathrm{~L}^{\prime 2+}$ complex (with $\mathrm{L}$ and $\mathrm{L}^{\prime}$ two different ligands), do not involve charge transfer to both $\mathrm{L}^{\prime}$ and $\mathrm{L}$, but to only one ligand, either $\mathrm{L}^{\prime}$ or $\mathrm{L}^{63}$

At energies higher than the triplet MLCT states, triplet states centered on ruthenium can be found, which are called metal-centered states $\left({ }^{3} \mathrm{MC}\right) .{ }^{95}$ These states are found at $\sim 3.3$ $\mathrm{eV}$ in the vertical excitations, which qualitatively agrees with what has been observed for the ground state eDoS (see Figure 7 ), showing contributions due to $\mathrm{Ru}(\mathrm{II})$ cations at energies higher than $3 \mathrm{eV}$ above the LUMO.

While singlet and triplet MLCT states have a relaxed geometry close to that of the ground state, relaxed ${ }^{3} \mathrm{MC}$ states show more significant distortions: there is a loss of coplanarity between the two rings of the ligands, with dihedral angles between the two aromatic subunits of more than $7^{\circ}$ (see Figure S6 (right pane) of the Supporting Information). This suggests that intersections with nearby excited states may occur, potentially leading to ISC phenomena.

Even if these triplet states are centered on the metal, they involve an increase in the positive Hirshfeld partial charge on the $\mathrm{Ru}(\mathrm{II})$ ion with respect to the ground state, as shown in Figure S8 of the Supporting Information. This can be explained as ${ }^{3} \mathrm{MC}$ states are thought to be predissociation states, $^{91,96-98}$ thus leaving a higher excess positive charge on the $\mathrm{Ru}(\mathrm{II})$ cation. While this latter increment is small in absolute value, in cases with a high content of nitrogen atoms, it represents an increase of about $40 \%$ in the overall positive charge on the cation, potentially changing the energetics of intermediate and transition states during catalytic processes going via the TTET mechanism.

4.3. Ground State Properties of Complexes Embedded into a COF. In second instance we investigate how the electronic properties of the ruthenium complexes are affected by embedding them into a CTF support. The CTF is composed of triazine nodes interlinked with bipy linkers and the bipy residues embedded into the CTF can naturally act as ligands for the ruthenium complexes. The fact that the ligand embedded into the CTF is bipy has also the effect of reducing the number of possible ligand combinations in the $\mathrm{RuL}_{2}{ }^{2+} @$ 
Scheme 2. Schematic Representation of Orbital Energies for the Ground State of Ru(bipy) ${ }_{2}^{2+} @$ CTF (Left Pane) and $\mathrm{Ru}(\mathrm{L})_{2}{ }^{2+} @ \mathrm{CTF}$ with 10 Nitrogen Atoms (Right Pane), Indicating the Proposed Change of Orbital Energies Due to an Increase of Nitrogen Content from 6 to 10 Atoms in the Ligands ${ }^{a}$

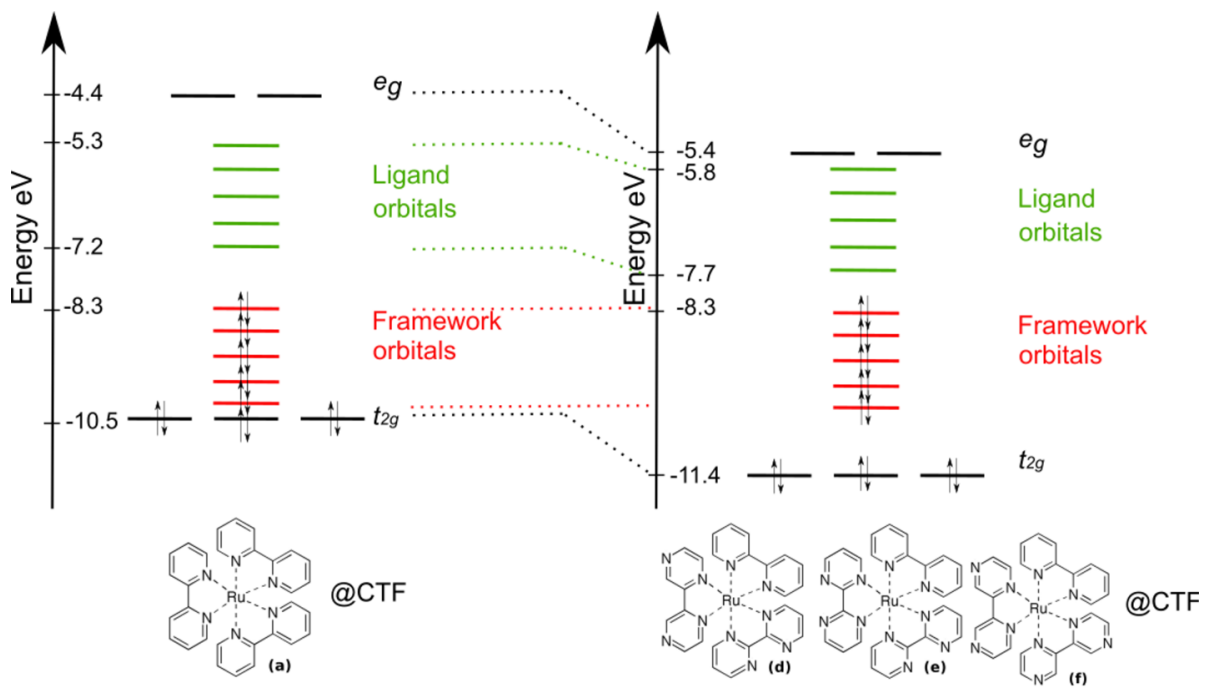

${ }^{a^{a}}$ The energy levels in the right pane correspond to Ru(bipz) ${ }_{2}{ }^{2+} @ \mathrm{CTF}$ and $\mathrm{Ru}(\text { bipzbipm })^{2+} @ \mathrm{CTF}$. Calculations are at the M06 level of theory.
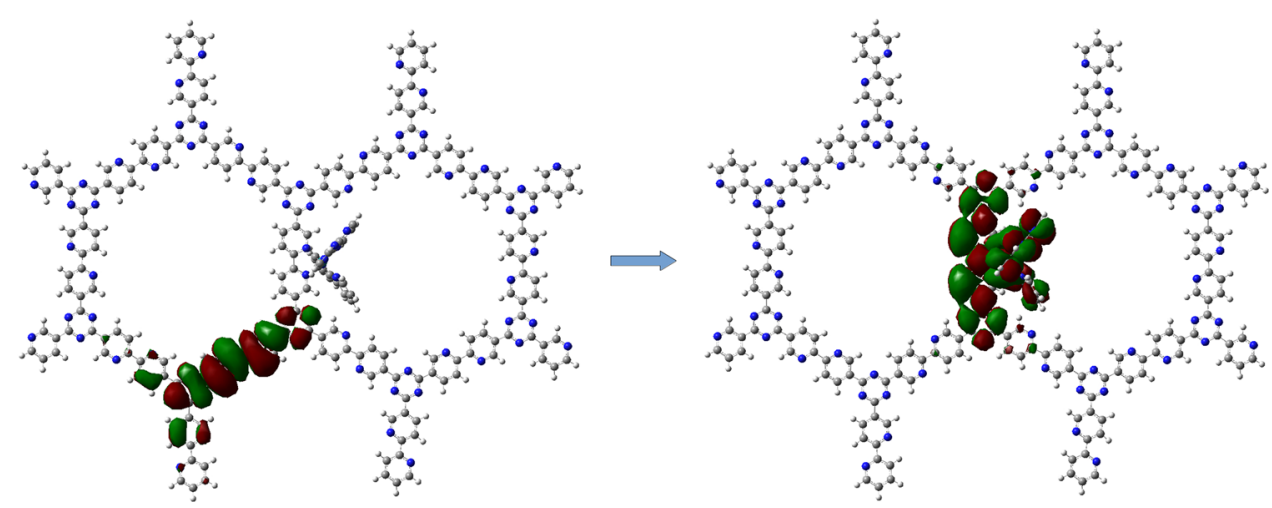

Figure 9. Orbitals involved in the first allowed transition in $\mathrm{Ru}(\text { bipy })_{2}{ }^{2+} @ \mathrm{CTF}$ complex. Calculation carried out at the M06/LanL2DZ level of theory.

CTF complex with respect to the isolated complexes from 10 to 6, i.e., complexes a-f in Figure 4.

We observe some significant electronic changes with respect to the isolated ruthenium complexes discussed before. In particular, occupied framework states are found between the states localized on the metal and on the ligands, as schematically shown in Scheme 2. This conclusion is in agreement with previous ground state calculations of $\mathrm{RuL}_{2}{ }^{2+} @$ CTF. ${ }^{63}$ According to the molecular orbital composition, the occupied $t_{2 g}$ and virtual $e_{g} d$ orbitals of the $\mathrm{Ru}(\mathrm{II})$ cation remain separated in energy by $\sim 6 \mathrm{eV}$ for the $\mathrm{Ru}(\text { bipy })_{2}{ }^{2+} @$ $\mathrm{CTF}$, which is close to the energy separation observed in the eDOS of the isolated complex (see Figure 6). However, in the $\mathrm{Ru} @ \mathrm{CTF}$ system, there are occupied framework states between the occupied orbitals of ruthenium and the virtual orbitals on the ligands, lowering the HOMO-LUMO gaps substantially by about $2.5-2.8 \mathrm{eV}$ compared to the isolated complexes.

Furthermore, we also investigate the influence of the nitrogen content on the electronic states of the system. The nitrogen content has a clear influence on the electronic states of the system, as schematically shown in the right pane of
Scheme 2. An increased nitrogen content from 6 to 10 atoms reduces the energy of $t_{2 g}$ and $e_{g}$ orbitals by about $1 \mathrm{eV}$ with respect to $\mathrm{Ru}(\text { bipy })_{2}{ }^{2+} @ \mathrm{CTF}$, the shift for Ru(bipm $)_{2}{ }^{2+} @ \mathrm{CTF}$ is somewhat smaller, i.e. $0.6 \mathrm{eV}$. Instead the energy of the framework orbitals $(\sim 8.3 \mathrm{eV})$ is rather unaffected. The virtual orbitals of the ligands are decreasing with about $0.5 \mathrm{eV}$, thus bringing the energy gap between HOMO and LUMO to values of the order of $0.6-0.8 \mathrm{eV}$ for nitrogen rich compounds such as $\mathrm{Ru}(\mathrm{bipz})_{2}{ }^{2+} @ \mathrm{CTF}, \mathrm{Ru}(\mathrm{bipm})_{2}{ }^{2+} @ \mathrm{CTF}$, and Ru(bipzbipm) $)^{2+} @$ CTF. The exact numerical values for the HOMO-LUMO gaps of the CTF-embedded complexes are reported in Table S6 of the Supporting Information.

4.4. Excited States Properties of Complexes Embedded into a COF. In CTF-embedded complexes, we notice that the orbital character of the first allowed TD M06 excitation, i.e., the first excitation with nonvanishing oscillator strength, is no longer mainly of the MLCT type as was the case for isolated complexes. Instead, we observe a charge transfer from states localized on the organic framework toward states localized on the complex, as shown in Figure 9. The target orbital is mainly localized on the bipyridine ligand of the CTF and only marginally on the ligands exposed inside the pore. 
These orbitals are the LUMOs in case of $\mathrm{Ru}(\text { bipy })_{2}{ }^{2+} @ \mathrm{CTF}$. It may be possible that if the pore ligands should have a higher nitrogen content, the target orbital would be situated on them instead, as ground state calculations on similar periodic systems suggest. ${ }^{63}$

The first allowed transition in $\mathrm{RuL}_{2}{ }^{2+} @ \mathrm{CTF}$ is thus of the crystal to crystal charge transfer (CCCT) or crystal to ligand charge transfer (CLCT) type. In the work of De Vos et al., where periodic ground state electronic structures were performed on the ruthenium complexes in CTF frameworks, it was indeed suggested that excitations of the highest occupied crystal orbital to the linkers or ligands attached to the ruthenium ion might be interesting, but it could not be deduced whether those states would be realistic as they are spatially relatively far separated. Here we find based on excited state calculations that these transitions are indeed realistic as they have a nonvanishing oscillator strength. A list of all excitation energies and their corresponding oscillator strengths is given in Table S7 in the Supporting Information.

Changing the nitrogen content of the ligands connected to ruthenium and pointing into the pores of the material, induces a significant drop in the vertical excitation energy of the most intense transition, with values as low as $1.6 \mathrm{eV}$ for an increasing number of nitrogen atoms in the ligands around ruthenium (see Figure 10). So, while in isolated complexes the excitation

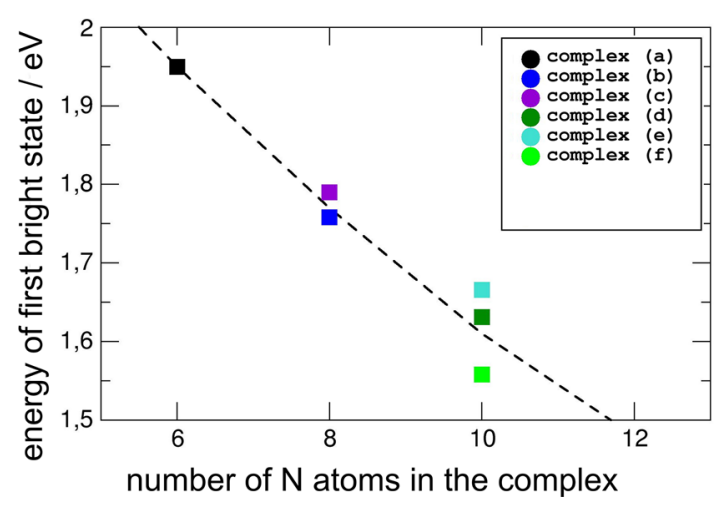

Figure 10. Change of the strongest excitation energy with the number of $\mathrm{N}$ atoms in the ligands around the $\mathrm{Ru}(\mathrm{II})$ cation in $\mathrm{RuL}_{2}{ }^{2+} @ \mathrm{CTF}$. The six RuL ${ }_{2}^{2+} @ \mathrm{CTF}$ complexes are (a) L = bipy $\times 2$; (b) $\mathrm{L}=$ bipy $\times$ 1 , bipz $\times 1$; (c) $\mathrm{L}=$ bipy $\times 1$, bipm $\times 1$; (d) L = bipz $\times 1$, bipm $\times 1$; (e) $\mathrm{L}=\operatorname{bipm} \times 2$; (f) $\mathrm{L}=\operatorname{bipz} \times 2$. Calculation carried out at the M06/LanL2DZ level of theory.

energy of the strongest transition is in the UV/blue visible region, the gap falls into the orange-near-infrared (NIR) region when the complex is included into the CTF (see Figure 11). Furthermore, it has to be emphasized that this effect is specific to this metal complex@CTF adduct, as the optical gap of the
CTF model alone (i.e., without any anchored ruthenium complex) is $\sim 3.32 \mathrm{eV}$, which is close to the near-UV range of energies instead.

While in isolated complexes the change of the excitation energy of the strongest transition with the number of nitrogen atoms of the ligands was small and nonmonotonous, a clear decrease of the excitation energy with the nitrogen content is observed in the embedded complexes (see Figure 10). This is due to the fact that the HOMO is no longer localized on the ruthenium but on the CTF, whereas the target virtual orbital is mainly localized on the ligands of the ruthenium complex.

These results show that the hybrid $\mathrm{RuL}_{2}{ }^{2+} @ \mathrm{CTF}$ system offers an extra degree of versatility in tuning the electronic response of the photocatalyst. By tuning the nitrogen content of the anchoring linkers pointing into the pore of the $\mathrm{RuL}_{2}{ }^{2+} @$ CTF system, the optical gap can be reduced by about $0.5 \mathrm{eV}$ and the direction of the charge transfer can be designed. A schematic representation of the excitation energies corresponding to the strongest transition in both isolated, embedded ruthenium complex and isolated CTF is shown in Figure 11 together with an indication on how these excitations would be influenced by the nitrogen content.

As it is known that TD-DFT might fail to correctly reproduce charge-transfer excitations, ${ }^{99}$ it is important to corroborate the above interpretation with ground state considerations. We notice here that the predicted TD M06 optical gaps shown in Figure 10 are in qualitative agreement with those of the occupied framework orbitals/virtual ligand orbitals described in Scheme 2 calculated with ground state M06 calculations. Furthermore, the trend with the nitrogen content is similar. Moreover, to further assess the reliability of the previous M06 ground state considerations, we have employed the range-separated CAM-B3LYP exchange correlation functional (which is often considered better suited to describe charge-transfer phenomena ${ }^{100}$ ) to compute KohnSham gaps in the isolated and CTF-embedded complexes. In this case, the gap between the occupied framework orbitals and virtual ligand orbitals calculated at the CAM-B3LYP level of theory is about $1.9-1.7 \mathrm{eV}$ smaller than the HOMO-LUMO gap in isolated complexes, which is in qualitative agreement with the aforementioned M06 results. With CAM-B3LYP, however, all the absolute energies are significantly blueshifted. This seems reasonable, as range-separated hybrid functionals are outperformed by global hybrid functionals for $\mathrm{n} \rightarrow \pi^{*}$ transitions. $^{64}$ To further investigate the nature of this transition, we have used the Mulliken averaged configuration $\left(M_{\mathrm{AC}}\right)$ index of Ciofini and co-workers that is able to spot ghost states which appear by significantly underestimating the energy. ${ }^{101}$ This diagnostic index, which discerns unrealistic charge transfer produced by the limitations of the level of theory, is a generalization of the Mulliken estimation of

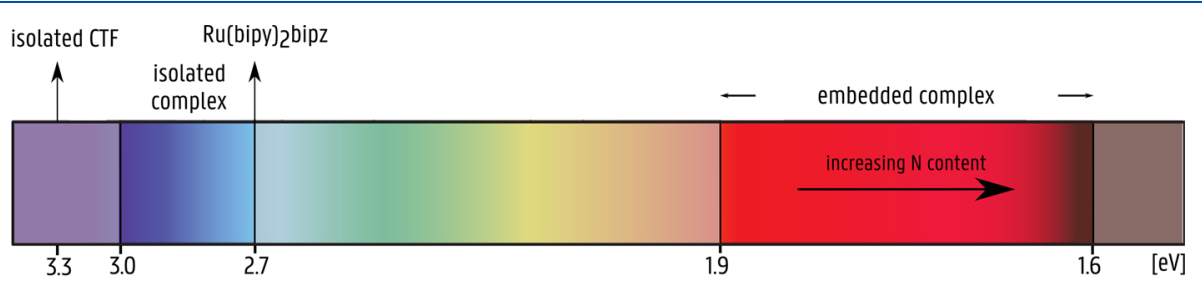

Figure 11. Schematic representation of the strongest singlet excitation energies of the isolated and embedded complexes. The lowest excitation energy for the isolated complex is found for $\mathrm{Ru}(\text { bipy })_{2} \mathrm{bipz}$ at $\sim 2.7 \mathrm{eV}$. For the embedded complexes the excitation energy decreases for an increasing number of nitrogen atoms. The energy of the first transition with nonzero oscillator strength of the empty CTF is also indicated. 
transition energy for charge transfer excitations. With it we were able to confirm the charge transfer character of the transition; it is not a ghost state as the transition energy is larger than the $M_{\mathrm{AC}}$ index. From this, we are ensured that our TD-DFT results are reliable.

\section{CONCLUSIONS}

In this work, we have investigated how polypyridyl ligands, characterized by a varying nitrogen content and aromaticity, can affect the photocatalytic properties of ruthenium-based complexes of the type $\mathrm{RuL}_{3}{ }^{2+}$ in both the ground and the excited states. As those complexes can be exploited either as oxidant or reductant agents in photochemical reactions, this overall insight into their energetics allows a choice for the most appropriate compound for the specific task at hand. Studying triplet states with computational approaches is necessary to optimize catalytic conditions for reactions going through triplet-triplet energy transfer (TTET), since investigating nonsinglet states by means of UV-vis absorption is often unpractical.

In second instance, we studied how the heterogenization of the ruthenium complexes on a CTF support consisting of bipyridine ligands affects the electronic ground and excited state properties of the $\mathrm{RuL}_{2}{ }^{2+} @ \mathrm{CFT}$ system.

For the isolated $\mathrm{RuL}_{3}{ }^{2+}$, an increase in the number of nitrogen atoms in the ligands redshifts the overall energetics of the compound, albeit without significantly altering the HOMO-LUMO gap. The energy gaps between linker-linker states, on the other hand, are significantly affected by both aromaticity and nitrogen content of the ligands. Singlet metalto-ligand charge transfer (MLCT) excitations are found between 2.2 and $2.6 \mathrm{eV}$ for complexes within varying nitrogen content. They are only slightly affected by changing the ligands. Triplet metal-to-ligand charge transfer (MLCT) excitations are situated in the $1.5-1.8 \mathrm{eV}$ range and can to a certain extent be tuned for a range of applications that require specific activation energies. One particular complex, $\mathrm{Ru}-$ (bipy) ${ }_{2}(\text { bipz })^{2+}$, shows a redshift of both singlet and ${ }^{3} \mathrm{MLCT}$ triplet excited states, making it an interesting candidate for processes induced by visible light. Changes induced in higherenergy triplet metal-centered (MC) states moreover deplete the electronic density around the $\mathrm{Ru}(\mathrm{II})$ cation, increasing its positive charge and, thus, its oxidant power.

For the complex embedded into a bipyridine-composed CTF, we observe a significant redshift of the allowed excitations to energies bordering the near IR region of the spectrum. This allows harvesting these lower energies to promote charge-transfer excitations. For example, near-infrared photocatalysis ${ }^{102,103}$ could greatly benefit from the nanoporous environment and thus make better use of the near-infrared solar irradiation (making up 44\% of solar irradiation spectrum) to reduce the ruthenium active site. Furthermore, the first allowed transition occurs from orbitals localized on the framework toward orbitals mainly localized on the ruthenium complex.

Overall, this investigation indicates how optoelectronic properties of ruthenium complexes can be changed and, possibly, tuned by the ligands and heterogenization within a CTF environment. Furthermore, this means that, potentially, a larger spectrum of radiation can be harvested to activate the complex. In the future, this kind of investigation could also be extended to other nitrogen containing aromatic ligands and transition metals, e.g., iridium, to further develop these promising platforms for heterogeneous photocatalysis.

\section{ASSOCIATED CONTENT}

\section{S Supporting Information}

The Supporting Information is available free of charge on the ACS Publications website at DOI: 10.1021/acs.jpca.9b05216.

Charges, spectra and optimized structures of $\mathrm{Ru}$ (bipy) ${ }_{3}{ }^{2+}$ across different functionals; ground state orbital isosurfaces of $\mathrm{Ru}(\mathrm{bipm})_{3}{ }^{2+}$; atomic contributions and energies of $\mathrm{Ru}(\text { bipy })_{3}{ }^{2+}$; influence of polypyridyl aromaticity on the electronic levels; comparison of charge schemes; excitation energies and oscillator strengths of isolated and embedded $\mathrm{Ru}$ (II) polypyridyl complexes; influence of excited-state optimization; removal of triplet state degeneracy; and $\mathrm{Ru}$ charge in metal-centered states (PDF)

\section{AUTHOR INFORMATION}

\section{Corresponding Authors}

*(K.L.) E-mail: kurt.lejaeghere@ugent.be.

*(V.V.S.) E-mail: Veronique.VanSpeybroeck@UGent.be. ORCID $\odot$

Arthur De Vos: 0000-0002-4916-6066

Christian V. Stevens: 0000-0003-4393-5327

Pascal Van Der Voort: 0000-0002-4874-0943

Kurt Lejaeghere: 0000-0002-4462-8209

Veronique Van Speybroeck: 0000-0003-2206-178X

Present Address

${ }^{\perp}$ Chimie ParisTech, PSL Research University, CNRS, Institute of Chemistry for Life and Health Sciences (i-CLeHS), FRE 2027, F-75005 Paris, France

\section{Author Contributions}

"These authors contributed equally

Notes

The authors declare no competing financial interest.

\section{ACKNOWLEDGMENTS}

The authors acknowledge the Fund for Scientific Research Flanders (FWO) and the Research Board of Ghent University (BOF) through a Concerted Research Action (GOA) for funding. V.V.S. and L.D.B. acknowledge funding from the European Union's Horizon 2020 research and innovation program (consolidator ERC Grant Agreement No. 647755 DYNPOR (2015-2020)). The computational resources and services used in this work were provided by VSC (Flemish Supercomputer Center), funded by Ghent University, FWO, and the Flemish Government Department EWI.

\section{REFERENCES}

(1) Armor, J. N. A History of Industrial Catalysis. Catal. Today 2011, 163, 3-9.

(2) Editorial. Closing in on Catalysis. Nat. Chem. Biol. 2009, 5, 515515

(3) Prier, C. K.; Rankic, D. A.; MacMillan, D. W. C. Visible Light Photoredox Catalysis with Transition Metal Complexes: Applications in Organic Synthesis. Chem. Rev. 2013, 113, 5322-5363.

(4) Kärkäs, M. D.; Porco, J. A.; Stephenson, C. R. J. Photochemical Approaches to Complex Chemotypes: Applications in Natural Product Synthesis. Chem. Rev. 2016, 116, 9683-9747.

(5) Slater, A. G.; Cooper, A. I. Porous Materials. Function-Led Design of New Porous Materials. Science 2015, 348, aaa8075. 
(6) Rogge, S. M. J.; Bavykina, A.; Hajek, J.; Garcia, H.; OlivosSuarez, A. I.; Sepúlveda-Escribano, A.; Vimont, A.; Clet, G.; Bazin, P.; Kapteijn, F.; et al. Metal-Organic and Covalent Organic Frameworks as Single-Site Catalysts. Chem. Soc. Rev. 2017, 46, 3134-3184.

(7) Takeda, H.; Ohashi, M.; Goto, Y.; Ohsuna, T.; Tani, T.; Inagaki, S. A Versatile Solid Photosensitizer: Periodic Mesoporous Organosilicas with Ruthenium Tris(Bipyridine) Complexes Embedded in the Pore Walls. Adv. Funct. Mater. 2016, 26, 5068-5077.

(8) Tu, W.; Xu, Y.; Yin, S.; Xu, R. Rational Design of Catalytic Centers in Crystalline Frameworks. Adv. Mater. 2018, 30, 1707582.

(9) Hug, S.; Tauchert, M. E.; Li, S.; Pachmayr, U. E.; Lotsch, B. V. A Functional Triazine Framework Based on N-Heterocyclic Building Blocks. J. Mater. Chem. 2012, 22, 13956-13964.

(10) Hedstrand, D.; Kruizinga, W.; Kellogg, R. Light-Induced and Dye Accelerated Reductions of Phenacyl Onium Salts by 1,4Dihydropyridines. Tetrahedron Lett. 1978, 19, 1255-1258.

(11) Takeda, H.; Ishitani, O. Development of Efficient Photocatalytic Systems for $\mathrm{CO}_{2}$ Reduction Using Mononuclear and Multinuclear Metal Complexes Based on Mechanistic Studies. Coord. Chem. Rev. 2010, 254, 346-354.

(12) Sekizawa, K.; Sato, S.; Arai, T.; Morikawa, T. Solar-Driven Photocatalytic $\mathrm{CO}_{2}$ Reduction in Water Utilizing a Ruthenium Complex Catalyst on p-Type $\mathrm{Fe}_{2} \mathrm{O}_{3}$ with a Multiheterojunction. ACS Catal. 2018, 8, 1405-1416.

(13) Gao, F.; Wang, Y.; Zhang, J.; Shi, D.; Wang, M.; HumphryBaker, R.; Wang, P.; Zakeeruddin, S. M.; Grätzel, M. A New Heteroleptic Ruthenium Sensitizer Enhances the Absorptivity of Mesoporous Titania Film for a High Efficiency Dye-Sensitized Solar Cell. Chem. Commun. 2008, 2635-2637.

(14) Kamata, R.; Kumagai, H.; Yamazaki, Y.; Sahara, G.; Ishitani, O. Photoelectrochemical $\mathrm{CO}_{2}$ Reduction Using a $\mathrm{Ru}(\mathrm{II})-\mathrm{Re}(\mathrm{I})$ Supramolecular Photocatalyst Connected to a Vinyl Polymer on a $\mathrm{NiO}$ Electrode. ACS Appl. Mater. Interfaces 2019, 11, 5632-5641.

(15) Concepcion, J. J.; Jurss, J. W.; Templeton, J. L.; Meyer, T. J. One Site Is Enough. Catalytic Water Oxidation by $[\mathrm{Ru}(\mathrm{Tpy})(\mathrm{Bpm})$ $\left.\left(\mathrm{OH}_{2}\right)\right]^{2+}$ and $\left[\mathrm{Ru}(\mathrm{Tpy})(\mathrm{Bpz})\left(\mathrm{OH}_{2}\right)\right]^{2+}$. J. Am. Chem. Soc. 2008, 130, 16462-16463.

(16) Kaveevivitchai, N.; Chitta, R.; Zong, R.; El Ojaimi, M.; Thummel, R. P. A Molecular Light-Driven Water Oxidation Catalyst. J. Am. Chem. Soc. 2012, 134, 10721-10724.

(17) Gil-Sepulcre, M.; Böhler, M.; Schilling, M.; Bozoglian, F.; Bachmann, C.; Scherrer, D.; Fox, T.; Spingler, B.; Gimbert-Suriñach, C.; Alberto, R.; et al. Ruthenium Water Oxidation Catalysts Based on Pentapyridyl Ligands. ChemSusChem 2017, 10, 4517-4525.

(18) Lin, S.; Ischay, M. A.; Fry, C. G.; Yoon, T. P. Radical Cation Diels-Alder Cycloadditions by Visible Light Photocatalysis. J. Am. Chem. Soc. 2011, 133, 19350-19353.

(19) Matsui, K.; Shibuya, M.; Yamamoto, Y. Synthesis of Pyrroles via Ruthenium-Catalyzed Nitrogen-Transfer $[2+2+1]$ Cycloaddition of $\alpha, \omega$-Diynes Using Sulfoximines as Nitrene Surrogates. Commun. Chem. 2018, 1, 21.

(20) Karges, J.; Heinemann, F.; Maschietto, F.; Patra, M.; Blacque, O.; Ciofini, I.; Spingler, B.; Gasser, G. A Ru(II) Polypyridyl Complex Bearing Aldehyde Functions as a Versatile Synthetic Precursor for Long-Wavelength Absorbing Photodynamic Therapy Photosensitizers. Bioorg. Med. Chem. 2019, 27, 2666-2675.

(21) Ghizdavu, L.; Pierard, F.; Rickling, S.; Aury, S.; Surin, M.; Beljonne, D.; Lazzaroni, R.; Murat, P.; Defrancq, E.; Moucheron, C.; et al. Oxidizing $\mathrm{Ru}(\mathrm{II})$ Complexes as Irreversible and Specific PhotoCross-Linking Agents of Oligonucleotide Duplexes. Inorg. Chem. 2009, 48, 10988-10994.

(22) Kalyanasundaram, K. Photophysics, Photochemistry and Solar Energy Conversion with Tris(Bipyridyl)Ruthenium(II) and Its Analogues. Coord. Chem. Rev. 1982, 46, 159-244.

(23) Estalayo-Adrián, S.; Garnir, K.; Moucheron, C. Perspectives of Ruthenium(Ii) Polyazaaromatic Photo-Oxidizing Complexes Photoreactive towards Tryptophan-Containing Peptides and Derivatives. Chem. Commun. 2018, 54, 322-337.
(24) Kulkarni, M. S.; Kumbhar, A. S.; Mohan, H.; Rao, B. S. M. Synthesis, Characterization and Redox Chemistry of $\mathrm{Ru}(\mathrm{II})$ Complexes of N-Methyl Pyridyl Quinoxaline. Dalton Trans. 2009, 6185-6191.

(25) Nakada, A.; Koike, K.; Nakashima, T.; Morimoto, T.; Ishitani, O. Photocatalytic $\mathrm{CO}_{2}$ Reduction to Formic Acid Using a $\mathrm{Ru}(\mathrm{II})-$ $\operatorname{Re}(\mathrm{I})$ Supramolecular Complex in an Aqueous Solution. Inorg. Chem. 2015, 54, 1800-1807.

(26) Atkins, A. J.; González, L. Trajectory Surface-Hopping Dynamics Including Intersystem Crossing in $\left[\mathrm{Ru}(\mathrm{Bpy})_{3}\right]^{2+}$. J. Phys. Chem. Lett. 2017, 8, 3840-3845.

(27) Olivares-Amaya, R.; Hu, W.; Nakatani, N.; Sharma, S.; Yang, J.; Chan, G. K.-L. The Ab-Initio Density Matrix Renormalization Group in Practice. J. Chem. Phys. 2015, 142, 034102.

(28) Ho, J.; Kish, E.; Méndez-Hernández, D. D.; WongCarter, K.; Pillai, S.; Kodis, G.; Niklas, J.; Poluektov, O. G.; Gust, D.; Moore, T. A.; et al. Triplet-Triplet Energy Transfer in Artificial and Natural Photosynthetic Antennas. Proc. Natl. Acad. Sci. U. S. A. 2017, 114, E5513-E5521.

(29) Wrighton, M.; Markham, J. Quenching of the Luminescent State of Tris(2,2'-Bipyridine)Ruthenium(II) by Electronic Energy Transfer. J. Phys. Chem. 1973, 77, 3042-3044.

(30) Islangulov, R. R.; Castellano, F. N. Photochemical Upconversion: Anthracene Dimerization Sensitized to Visible Light by a $\mathrm{Ru}^{\mathrm{II}}$ Chromophore. Angew. Chem., Int. Ed. 2006, 45, 5957-5959.

(31) Lu, Z.; Yoon, T. P. Visible Light Photocatalysis of $[2+2]$ Styrene Cycloadditions via Energy Transfer. Angew. Chem., Int. Ed. 2012, 51, 10329-10332.

(32) Lin, Q.-Y.; Xu, X.-H.; Qing, F.-L. Chemo-, Regio-, and Stereoselective Trifluoromethylation of Styrenes via Visible LightDriven Single-Electron Transfer (SET) and Triplet-Triplet Energy Transfer (TTET) Processes. J. Org. Chem. 2014, 79, 10434-10446.

(33) Strouse, G. F.; Anderson, P. A.; Schoonover, J. R.; Meyer, T. J.; Keene, F. R. Synthesis of Polypyridyl Complexes of Ruthenium(II) Containing Three Different Bidentate Ligands. Inorg. Chem. 1992, 31, 3004-3006.

(34) Kubeil, M.; Vernooij, R. R.; Kubeil, C.; Wood, B. R.; Graham, B.; Stephan, H.; Spiccia, L. Studies of Carbon Monoxide Release from Ruthenium(II) Bipyridine Carbonyl Complexes upon UV-Light Exposure. Inorg. Chem. 2017, 56, 5941-5952.

(35) Myahkostupov, M.; Castellano, F. N. Synthesis and Characterization of Tris(Heteroleptic) $\mathrm{Ru}(\mathrm{II})$ Complexes Bearing Styryl Subunits. Inorg. Chem. 2011, 50, 9714-9727.

(36) Gonçalves, R. S. B.; de Oliveira, A. B. V.; Sindra, H. C.; Archanjo, B. S.; Mendoza, M. E.; Carneiro, L. S. A.; Buarque, C. D.; Esteves, P. M. Heterogeneous Catalysis by Covalent Organic Frameworks (COF): $\mathrm{Pd}(\mathrm{OAc}) 2 @ \mathrm{COF}-300$ in Cross-Coupling Reactions. Chem CatChem 2016, 8, 743-750.

(37) Vyas, V. S.; Haase, F.; Stegbauer, L.; Savasci, G.; Podjaski, F.; Ochsenfeld, C.; Lotsch, B. V. A Tunable Azine Covalent Organic Framework Platform for Visible Light-Induced Hydrogen Generation. Nat. Commun. 2015, 6, 8508.

(38) Stegbauer, L.; Schwinghammer, K.; Lotsch, B. V. A HydrazoneBased Covalent Organic Framework for Photocatalytic Hydrogen Production. Chem. Sci. 2014, 5, 2789-2793.

(39) Banerjee, T.; Lotsch, B. V. The Wetter the Better. Nat. Chem. 2018, 10, 1175-1177.

(40) Ding, S.-Y.; Wang, W. Covalent Organic Frameworks (COFs): From Design to Applications. Chem. Soc. Rev. 2013, 42, 548-568.

(41) Kuhn, P.; Antonietti, M.; Thomas, A. Porous, Covalent Triazine-Based Frameworks Prepared by Ionothermal Synthesis. Angew. Chem., Int. Ed. 2008, 47, 3450-3453.

(42) Feng, X.; Ding, X.; Jiang, D. Covalent Organic Frameworks. Chem. Soc. Rev. 2012, 41, 6010-6022.

(43) Stewart, D.; Antypov, D.; Dyer, M. S.; Pitcher, M. J.; Katsoulidis, A. P.; Chater, P. A.; Blanc, F.; Rosseinsky, M. J. Stable and Ordered Amide Frameworks Synthesised under Reversible Conditions Which Facilitate Error Checking. Nat. Commun. 2017, 8,1102 . 
(44) Halder, A.; Karak, S.; Addicoat, M.; Bera, S.; Chakraborty, A.; Kunjattu, S. H.; Pachfule, P.; Heine, T.; Banerjee, R. Ultrastable Imine-Based Covalent Organic Frameworks for Sulfuric Acid Recovery: An Effect of Interlayer Hydrogen Bonding. Angew. Chem., Int. Ed. 2018, 57, 5797-5802.

(45) Ding, S.-Y.; Gao, J.; Wang, Q.; Zhang, Y.; Song, W.-G.; Su, C.Y.; Wang, W. Construction of Covalent Organic Framework for Catalysis: Pd/COF-LZU1 in Suzuki-Miyaura Coupling Reaction. J. Am. Chem. Soc. 2011, 133, 19816-19822.

(46) Waller, P. J.; Gándara, F.; Yaghi, O. M. Chemistry of Covalent Organic Frameworks. Acc. Chem. Res. 2015, 48, 3053-3063.

(47) Liu, M.; Guo, L.; Jin, S.; Tan, B. Covalent Triazine Frameworks: Synthesis and Applications. J. Mater. Chem. A 2019, 7, 5153-5172.

(48) Hug, S.; Stegbauer, L.; Oh, H.; Hirscher, M.; Lotsch, B. V. Nitrogen-Rich Covalent Triazine Frameworks as High-Performance Platforms for Selective Carbon Capture and Storage. Chem. Mater. 2015, 27, 8001-8010.

(49) Park, K.; Gunasekar, G. H.; Prakash, N.; Jung, K.-D.; Yoon, S. A Highly Efficient Heterogenized Iridium Complex for the Catalytic Hydrogenation of Carbon Dioxide to Formate. ChemSusChem 2015, 8, 3410-3413.

(50) Tahir, N.; Muniz-Miranda, F.; Everaert, J.; Tack, P.; Heugebaert, T.; Leus, K.; Vincze, L.; Stevens, C.; Van Speybroeck, V.; Van Der Voort, P. Immobilization of $\operatorname{Ir}(\mathrm{I})$ Complex on Covalent Triazine Frameworks for CAH Borylation Reactions: A Combined Experimental and Computational Study. J. Catal. 2019, 371, 135143.

(51) Johnson, E. M.; Haiges, R.; Marinescu, S. C. Covalent-Organic Frameworks Composed of Rhenium Bipyridine and Metal Porphyrins: Designing Heterobimetallic Frameworks with Two Distinct Metal Sites. ACS Appl. Mater. Interfaces 2018, 10, 37919-37927.

(52) Aiyappa, H. B.; Thote, J.; Shinde, D. B.; Banerjee, R.; Kurungot, S. Cobalt-Modified Covalent Organic Framework as a Robust Water Oxidation Electrocatalyst. Chem. Mater. 2016, 28, 4375-4379.

(53) Popov, D. A.; Luna, J. M.; Orchanian, N. M.; Haiges, R.; Downes, C. A.; Marinescu, S. C. A 2,2'-Bipyridine-Containing Covalent Organic Framework Bearing Rhenium(i) Tricarbonyl Moieties for $\mathrm{CO}_{2}$ Reduction. Dalton Trans. Camb. Engl. 2003 2018, 47, 17450-17460.

(54) Gunasekar, G. H.; Park, K.; Jeong, H.; Jung, K.-D.; Park, K.; Yoon, S. Molecular $\mathrm{Rh}(\mathrm{III})$ and $\mathrm{Ir}(\mathrm{III})$ Catalysts Immobilized on Bipyridine-Based Covalent Triazine Frameworks for the Hydrogenation of $\mathrm{CO}_{2}$ to Formate. Catalysts 2018, 8, 295.

(55) Hendon, C. H.; Bonnefoy, J.; Quadrelli, E. A.; Canivet, J.; Chambers, M. B.; Rousse, G.; Walsh, A.; Fontecave, M.; MellotDraznieks, C. A Simple and Non-Destructive Method for Assessing the Incorporation of Bipyridine Dicarboxylates as Linkers within Metal-Organic Frameworks. Chem. - Eur. J. 2016, 22, 3713-3718.

(56) Maza, W. A.; Morris, A. J. Photophysical Characterization of a Ruthenium(II) Tris(2,2'-Bipyridine)-Doped Zirconium UiO-67 Metal-Organic Framework. J. Phys. Chem. C 2014, 118, 8803-8817. (57) Hou, C.-C.; Li, T.-T.; Cao, S.; Chen, Y.; Fu, W.-F. Incorporation of a $\left[\mathrm{Ru}(\mathrm{Dcbpy})(\mathrm{Bpy})_{2}\right]^{2+}$ Photosensitizer and a $\mathrm{Pt}$ (Dcbpy) $\mathrm{Cl}_{2}$ Catalyst into Metal-Organic Frameworks for Photocatalytic Hydrogen Evolution from Aqueous Solution. J. Mater. Chem. A 2015, 3, 10386-10394.

(58) Yang, D.; Odoh, S. O.; Wang, T. C.; Farha, O. K.; Hupp, J. T.; Cramer, C. J.; Gagliardi, L.; Gates, B. C. Metal-Organic Framework Nodes as Nearly Ideal Supports for Molecular Catalysts: NU-1000and UiO-66-Supported Iridium Complexes. J. Am. Chem. Soc. 2015, 137, 7391-7396.

(59) Wang, C.; Xie, Z.; deKrafft, K. E.; Lin, W. Doping MetalOrganic Frameworks for Water Oxidation, Carbon Dioxide Reduction, and Organic Photocatalysis. J. Am. Chem. Soc. 2011, 133, 13445-13454.

(60) Burtch, N. C.; Jasuja, H.; Walton, K. S. Water Stability and Adsorption in Metal-Organic Frameworks. Chem. Rev. 2014, 114, 10575-10612.
(61) Leus, K.; Bogaerts, T.; De Decker, J.; Depauw, H.; Hendrickx, K.; Vrielinck, H.; Van Speybroeck, V.; Van Der Voort, P. Systematic Study of the Chemical and Hydrothermal Stability of Selected "Stable" Metal Organic Frameworks. Microporous Mesoporous Mater. 2016, 226, 110-116.

(62) Daniel, C. Photochemistry and Photophysics of Transition Metal Complexes: Quantum Chemistry. Coord. Chem. Rev. 2015, 282-283, 19-32.

(63) De Vos, A.; Lejaeghere, K.; Muniz-Miranda, F.; Stevens, C. V.; Van der Voort, P.; Van Speybroeck, V. Electronic Properties of Heterogenized $\mathrm{Ru}(\mathrm{II})$ Polypyridyl Photoredox Complexes on Covalent Triazine Frameworks. J. Mater. Chem. A 2019, 7, 84338442.

(64) Jacquemin, D.; Perpète, E. A.; Vydrov, O. A.; Scuseria, G. E.; Adamo, C. Assessment of Long-Range Corrected Functionals Performance for $\mathrm{n} \rightarrow \mathrm{pi}^{*}$ Transitions in Organic Dyes. J. Chem. Phys. 2007, 127, 094102.

(65) Frisch, M. J.; Trucks, G. W.; Schlegel, H. B.; Scuseria, G. E.; Robb, M. A.; Cheeseman, J. R.; Scalmani, G.; Barone, V.; Petersson, G. A.; Nakatsuj, H.; et al. Gaussian 16, Revision B.01; Gaussian, Inc.: Wallingford, CT, 2016.

(66) Runge, E.; Gross, E. K. U. Density-Functional Theory for Time-Dependent Systems. Phys. Rev. Lett. 1984, 52, 997-1000.

(67) Casida, M. E.; Jamorski, C.; Casida, K. C.; Salahub, D. R. Molecular Excitation Energies to High-Lying Bound States from Time-Dependent Density-Functional Response Theory: Characterization and Correction of the Time-Dependent Local Density Approximation Ionization Threshold. J. Chem. Phys. 1998, 108, 4439-4449.

(68) Perdew, J. P.; Ruzsinszky, A.; Tao, J.; Staroverov, V. N.; Scuseria, G. E.; Csonka, G. I. Prescription for the Design and Selection of Density Functional Approximations: More Constraint Satisfaction with Fewer Fits. J. Chem. Phys. 2005, 123, 062201.

(69) Perdew, J. P.; Burke, K.; Ernzerhof, M. Generalized Gradient Approximation Made Simple. Phys. Rev. Lett. 1996, 77, 3865-3868.

(70) Lee, C.; Yang, W.; Parr, R. G. Development of the ColleSalvetti Correlation-Energy Formula into a Functional of the Electron Density. Phys. Rev. B: Condens. Matter Mater. Phys. 1988, 37, 785789.

(71) Becke, A. D. Density-functional Thermochemistry. III. The Role of Exact Exchange. J. Chem. Phys. 1993, 98, 5648-5652.

(72) Yanai, T.; Tew, D. P.; Handy, N. C. A New Hybrid ExchangeCorrelation Functional Using the Coulomb-Attenuating Method (CAM-B3LYP). Chem. Phys. Lett. 2004, 393, 51-57.

(73) Zhao, Y.; Truhlar, D. G. The M06 Suite of Density Functionals for Main Group Thermochemistry, Thermochemical Kinetics, Noncovalent Interactions, Excited States, and Transition Elements: Two New Functionals and Systematic Testing of Four M06-Class Functionals and 12 Other Functionals. Theor. Chem. Acc. 2008, 120, 215-241.

(74) Adamo, C.; Jacquemin, D. The Calculations of Excited-State Properties with Time-Dependent Density Functional Theory. Chem. Soc. Rev. 2013, 42, 845-856.

(75) Tomasi, J.; Mennucci, B.; Cammi, R. Quantum Mechanical Continuum Solvation Models. Chem. Rev. 2005, 105, 2999-3094.

(76) Word, T. A.; Whittington, C. L.; Karolak, A.; Kemp, M. T.; Woodcock, H. L.; van der Vaart, A.; Larsen, R. W. Photoacoustic Calorimetry Study of Ligand Photorelease from the $\mathrm{Ru}(\mathrm{II}) \operatorname{Bis}\left(2,2^{\prime}\right.$ Bipyridine) (6,6'-Dimethyl-2,2'-Bipyridine) Complex in Aqueous Solution. Chem. Phys. Lett. 2015, 619, 214-218.

(77) Baerends, E. J.; Gritsenko, O. V.; van Meer, R. The KohnSham Gap, the Fundamental Gap and the Optical Gap: The Physical Meaning of Occupied and Virtual Kohn-Sham Orbital Energies. Phys. Chem. Chem. Phys. 2013, 15, 16408-16425.

(78) Kronik, L.; Stein, T.; Refaely-Abramson, S.; Baer, R. Excitation Gaps of Finite-Sized Systems from Optimally Tuned Range-Separated Hybrid Functionals. J. Chem. Theory Comput. 2012, 8, 1515-1531.

(79) Muniz-Miranda, F.; Menziani, M. C.; Pedone, A. DFT and TDDFT Assessment of the Structural and Optoelectronic Properties of 
an Organic $-\mathrm{Ag}_{14}$ Nanocluster. J. Phys. Chem. A 2015, 119, 50885098.

(80) Muniz-Miranda, F.; Menziani, M. C.; Pedone, A. Assessment of Exchange-Correlation Functionals in Reproducing the Structure and Optical Gap of Organic-Protected Gold Nanoclusters. J. Phys. Chem. C 2014, 118, 7532-7544.

(81) De Meyer, T.; Steyaert, I.; Hemelsoet, K.; Hoogenboom, R.; Van Speybroeck, V.; De Clerck, K. Halochromic Properties of Sulfonphthaleine Dyes in a Textile Environment: The Influence of Substituents. Dyes Pigm. 2016, 124, 249-257.

(82) Hendrickx, K.; Vanpoucke, D. E. P.; Leus, K.; Lejaeghere, K.; Van Yperen-De Deyne, A.; Van Speybroeck, V.; Van Der Voort, P.; Hemelsoet, K. Understanding Intrinsic Light Absorption Properties of UiO-66 Frameworks: A Combined Theoretical and Experimental Study. Inorg. Chem. 2015, 54, 10701-10710.

(83) Hay, P. J.; Wadt, W. R. Ab Initio Effective Core Potentials for Molecular Calculations. Potentials for the Transition Metal Atoms Sc to Hg. J. Chem. Phys. 1985, 82, 270-283.

(84) Petit, L.; Maldivi, P.; Adamo, C. Predictions of Optical Excitations in Transition-Metal Complexes with Time DependentDensity Functional Theory: Influence of Basis Sets. J. Chem. Theory Comput. 2005, 1, 953-962.

(85) Laurent, A. D.; Jacquemin, D. TD-DFT Benchmarks: A Review. Int. J. Quantum Chem. 2013, 113, 2019-2039.

(86) Jacquemin, D.; Perpète, E. A.; Ciofini, I.; Adamo, C.; Valero, R.; Zhao, Y.; Truhlar, D. G. On the Performances of the M06 Family of Density Functionals for Electronic Excitation Energies. J. Chem. Theory Comput. 2010, 6, 2071-2085.

(87) Atkins, A. J.; Talotta, F.; Freitag, L.; Boggio-Pasqua, M.; González, L. Assessing Excited State Energy Gaps with TimeDependent Density Functional Theory on Ru(II) Complexes. J. Chem. Theory Comput. 2017, 13, 4123-4145.

(88) Bird, C. W. Heteroaromaticity, 5, a Unified Aromaticity Index. Tetrahedron 1992, 48, 335-340.

(89) Bird, C. W. The Relationship of Classical and Magnetic Criteria of Aromaticity. Tetrahedron 1996, 52, 9945-9952.

(90) Wiberg, K. B.; Rablen, P. R. Comparison of Atomic Charges Derived via Different Procedures. J. Comput. Chem. 1993, 14, 15041518.

(91) Innocenzi, P.; Kozuka, H.; Yoko, T. Fluorescence Properties of the $\mathrm{Ru}(\mathrm{Bpy})_{3}{ }^{2+}$ Complex Incorporated in Sol-Gel-Derived Silica Coating Films. J. Phys. Chem. B 1997, 101, 2285-2291.

(92) Juris, A.; Balzani, V.; Belser, P.; von Zelewsky, A. Characterization of the Excited State Properties of Some New Photosensitizers of the Ruthenium (Polypyridine) Family. Helv. Chim. Acta 1981, 64, 2175-2182.

(93) Alary, F.; Boggio-Pasqua, M.; Heully, J. L.; Marsden, C. J.; Vicendo, P. Theoretical Characterization of the Lowest Triplet Excited States of the Tris-(1,4,5,8-Tetraazaphenanthrene) Ruthenium Dication Complex. Inorg. Chem. 2008, 47, 5259-5266.

(94) Alary, F.; Heully, J.-L.; Bijeire, L.; Vicendo, P. Is the ${ }^{3}$ MLCT the Only Photoreactive State of Polypyridyl Complexes? Inorg. Chem. 2007, 46, 3154-3165.

(95) Mukuta, T.; Tanaka, S.; Inagaki, A.; Koshihara, S. Y.; Onda, K. Direct Observation of the Triplet Metal-Centered State in [Ru(bpy) $\left.)_{3}\right]^{2+}$ Using Time-Resolved Infrared Spectroscopy. ChemistrySelect 2016, 1, 2802-2807.

(96) Alcover-Fortuny, G.; Wu, J.; Caballol, R.; de Graaf, C. Quantum Chemical Study of the Interligand Electron Transfer in $\mathrm{Ru}$ Polypyridyl Complexes. J. Phys. Chem. A 2018, 122, 1114-1123.

(97) Lord, R. L.; Allard, M. M.; Thomas, R. A.; Odongo, O. S.; Schlegel, H. B.; Chen, Y.-J.; Endicott, J. F. Computational Modeling of the Triplet Metal-to-Ligand Charge-Transfer Excited-State Structures of Mono-Bipyridine-Ruthenium(II) Complexes and Comparisons to Their $77 \mathrm{~K}$ Emission Band Shapes. Inorg. Chem. 2013, 52, 1185-1198.

(98) Österman, T.; Abrahamsson, M.; Becker, H.-C.; Hammarström, L.; Persson, P. Influence of Triplet State Multidimensionality on
Excited State Lifetimes of Bis-Tridentate RuII Complexes: A Computational Study. J. Phys. Chem. A 2012, 116, 1041-1050.

(99) Dreuw, A.; Head-Gordon, M. Failure of Time-Dependent Density Functional Theory for Long-Range Charge-Transfer Excited States: The Zincbacteriochlorin-Bacteriochlorin and Bacteriochlorophyll-Spheroidene Complexes. J. Am. Chem. Soc. 2004, 126, 40074016.

(100) Kobayashi, R.; Amos, R. Erratum to 'The Application of CAM-B3LYP to the Charge-Transfer Band Problem of the Zincbacteriochlorin-Bacteriochlorin Complex' [Chem. Phys. Lett. 420 (2006) 106-109]. Chem. Phys. Lett. 2006, 424, 225.

(101) Campetella, M.; Maschietto, F.; Frisch, M. J.; Scalmani, G.; Ciofini, I.; Adamo, C. Charge Transfer Excitations in TDDFT: A Ghost-Hunter Index. J. Comput. Chem. 2017, 38, 2151-2156.

(102) Zhang, L.; Wang, W.; Sun, S.; Jiang, D. Near-Infrared Light Photocatalysis with Metallic/Semiconducting $\mathrm{H}_{\mathrm{x}} \mathrm{WO}_{3} / \mathrm{WO}_{3}$ Nanoheterostructure in Situ Formed in Mesoporous Template. Appl. Catal., B 2015, 168, 9-13.

(103) Liu, W.-X.; Zhu, X.-L.; Liu, S.-Q.; Gu, Q.-Q.; Meng, Z.-D. Near-Infrared-Driven Selective Photocatalytic Removal of Ammonia Based on Valence Band Recognition of an $\alpha-\mathrm{MnO}_{2} / \mathrm{N}$-Doped Graphene Hybrid Catalyst. ACS Omega 2018, 3, 5537-5546. 